Supporting Information for:

\title{
A Highly Efficient and Direct Approach for Synthesis of Enantiopure ß-Amino Alcohols by Reductive Cross-Coupling of Chiral $N$-tert-Butanesulfinyl Imines with Aldehydes
}

Yu-Wu Zhong, Yi-Zhou Dong, Kai Fang, Kenji Izumi, Ming-Hua Xu, and Guo-Qiang Lin

Shanghai Institute of Organic Chemistry, Chinese Academy of Sciences, 354 Fenglin Road, Shanghai 200032, and Shanghai Institute of Materia Medica, Chinese Academy of Sciences, 555 Zuchongzhi Road, Shanghai 201203, China.

xumh@mail.sioc.ac.cn

\section{Table of Contents}

1. General procedure for the reductive cross-coupling of aldehydes with $N$-tert-butylsulfinyl imines.

2. Characterization of the obtained optically active $\beta$-amino alcohols. $. \mathrm{S} 2-\mathrm{S} 6$

3. Determination of the diastereoselectivity and the enantiomeric excess. $\mathrm{S} 6-\mathrm{S} 11$

4. Synthesis of D-erythro-sphinganine. $\mathrm{S} 11-\mathrm{S} 12$

5. Synthesis of $(3 R, 4 S)$-statine. 
1. General procedure for the reductive cross-coupling of aldehydes with $N$-tert-butylsulfinyl imines.

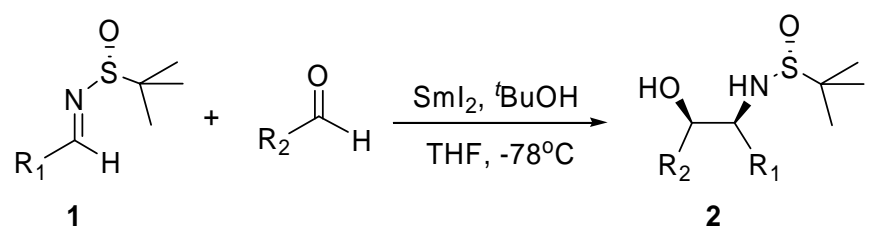

Under nitrogen, a freshly prepared solution of samarium diiodide $(1.0 \mathrm{mmol})$ in THF $(5 \mathrm{~mL})$ was cooled to $-78^{\circ} \mathrm{C}$. A mixture of $1.0 \mathrm{mmol}$ of tert-butyl alcohol, $0.75 \mathrm{mmol}$ of aldehyde, and $0.5 \mathrm{mmol}$ of chiral $N$-tert-butylsulfinyl imine in $6 \mathrm{~mL}$ of THF was added dropwise. (When $\mathrm{R}_{1}=4-\mathrm{FC}_{6} \mathrm{H}_{4}, 4-\mathrm{ClC}_{6} \mathrm{H}_{4}, 4-\mathrm{BrC}_{6} \mathrm{H}_{4}$ or $4-\mathrm{AcOC}_{6} \mathrm{H}_{4}$, a mixture of tert-butyl alcohol and aldehyde was first added, and $\mathrm{N}$-tert-butylsulfinyl imine was then added dropwise to minimize the homocoupling.) The reaction was monitored by TLC, and quenched with $5 \mathrm{~mL}$ of saturated aqueous $\mathrm{Na}_{2} \mathrm{~S}_{2} \mathrm{O}_{3}$ when the reaction goes to completion. Extraction with ethyl acetate and purification by flash column chromatography afforded the desired product.

\section{Characterization of the obtained optically active $\beta$-amino alcohols.}

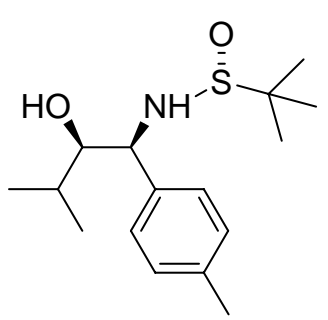

2a $[\alpha]_{\mathrm{D}}{ }^{20}=-48.2\left(c 0.80, \mathrm{CHCl}_{3}\right) ;{ }^{1} \mathrm{H} \mathrm{NMR}\left(300 \mathrm{MHz}, \mathrm{CDCl}_{3}\right)$ : $\delta 0.94(\mathrm{~d}, 3 \mathrm{H}, J=5.1 \mathrm{~Hz}), 0.96(\mathrm{~d}, 3 \mathrm{H}, J=5.1 \mathrm{~Hz}), 1.22(\mathrm{~s}, 9 \mathrm{H})$, $1.49(\mathrm{~m}, 1 \mathrm{H}), 2.07$ (br, 1H), $2.33(\mathrm{~s}, 3 \mathrm{H}), 3.61(\mathrm{br}, 1 \mathrm{H}), 3.68(\mathrm{~d}$, $1 \mathrm{H}, J=5.7 \mathrm{~Hz}), 4.45(\mathrm{t}, 1 \mathrm{H}, J=4.8 \mathrm{~Hz}), 7.14(\mathrm{~d}, 2 \mathrm{H}, J=8.1 \mathrm{~Hz})$, 7.29(d, 2H, $J=8.1 \mathrm{~Hz})$; FT-IR $\left(\mathrm{KBr}, \mathrm{cm}^{-1}\right): 3346,1463,1031$; ESI-MS (m/z, \%): $298.3\left(\mathrm{M}^{+}+\mathrm{H}\right) ;{ }^{13} \mathrm{C} \mathrm{NMR}\left(75 \mathrm{MHz}, \mathrm{CDCl}_{3}\right): \delta$ 18.26, 19.32, 21.09, 22.57, 30.17, 55.96, 60.42, 78.82, 128.39, 129.31, 135.39, 137.85; HRMS for $\mathrm{C}_{16} \mathrm{H}_{27} \mathrm{NO}_{2} \mathrm{SNa}\left(\mathrm{M}^{+}+\mathrm{Na}\right)$ : calcd. 320.1655 , found: 320.1634 .

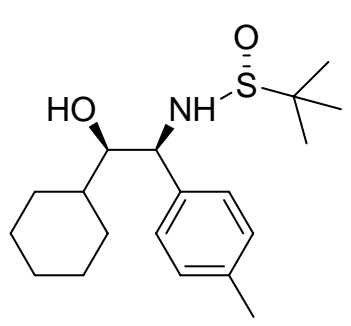

2b $[\alpha]_{\mathrm{D}}{ }^{20}=-53.8\left(c 1.35, \mathrm{CHCl}_{3}\right) ;{ }^{1} \mathrm{H} \mathrm{NMR}\left(300 \mathrm{MHz}, \mathrm{CDCl}_{3}\right)$ : $\delta 1.24(\mathrm{~d}, 6 \mathrm{H}, J=7.2 \mathrm{~Hz}), 1.24-1.26(\mathrm{~m}, 4 \mathrm{H}), 1.72-1.90(\mathrm{~m}$, $6 \mathrm{H}), 2.34(\mathrm{~s}, 3 \mathrm{H}), 3.66(\mathrm{dd}, 1 \mathrm{H}, J=4.2,7.2 \mathrm{~Hz}), 3.77$ (d, $1 \mathrm{H}, J$ $=5.7 \mathrm{~Hz}), 4.47(\mathrm{t}, 1 \mathrm{H}, J=4.9 \mathrm{~Hz}), 7.15(\mathrm{~d}, 2 \mathrm{H}, J=8.0 \mathrm{~Hz})$, $7.28(\mathrm{~d}, 2 \mathrm{H}, J=8.0 \mathrm{~Hz})$; FT-IR $\left(\mathrm{KBr}, \mathrm{cm}^{-1}\right): 3346,1463,1031$; ESI-MS (m/z, \%): $338\left(\mathrm{M}^{+}+\mathrm{H}\right) ;{ }^{13} \mathrm{C}$ NMR $\left(75 \mathrm{MHz}, \mathrm{CDCl}_{3}\right): \delta$ 21.12, 22.63, 25.59, 25.86, 26.33, 28.27, 29.55, 39.67, 56.03, 60.00, 78.05, 128.38, 129.36, 135.56, 137.80; HRMS for $\mathrm{C}_{19} \mathrm{H}_{31} \mathrm{NO}_{2} \mathrm{SNa}\left(\mathrm{M}^{+}+\mathrm{Na}\right)$ : calcd. 360.1967, found: 360.1974 .

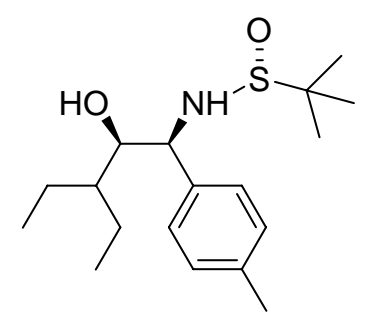

2c $[\alpha]_{\mathrm{D}}{ }^{20}=-36.0\left(\right.$ c 1.05, $\left.\mathrm{CHCl}_{3}\right) ;{ }^{1} \mathrm{H}$ NMR $\left(300 \mathrm{MHz}, \mathrm{CDCl}_{3}\right)$ : $\delta 0.85(\mathrm{q}, 6 \mathrm{H}, J=3.0 \mathrm{~Hz}), 1.19(\mathrm{~s}, 9 \mathrm{H}), 1.21-1.56(\mathrm{~m}, 5 \mathrm{H})$, $1.76(\mathrm{br}, 1 \mathrm{H}), 2.33(\mathrm{~s}, 3 \mathrm{H}), 3.64(\mathrm{~d}, 1 \mathrm{H}, J=5.4 \mathrm{~Hz}), 3.85$ (br, $1 \mathrm{H}$ ), $4.45(\mathrm{t}, 1 \mathrm{H}, J=5.4 \mathrm{~Hz}), 7.15(\mathrm{~d}, 2 \mathrm{H}, J=8.1 \mathrm{~Hz}), 7.28(\mathrm{~d}$, $2 \mathrm{H}, J=8.1 \mathrm{~Hz})$; FT-IR $\left(\mathrm{KBr}, \mathrm{cm}^{-1}\right): 3370,3336,2967,1513$, 
1466, 1033; ESI-MS (m/z, \%): $326.3\left(\mathrm{M}^{+}+\mathrm{H}\right) ;{ }^{13} \mathrm{C}$ NMR $\left(75 \mathrm{MHz}, \mathrm{CDCl}_{3}\right): \delta 10.50$, 10.61, 20.01, 21.10, 21.29, 22.59, 41.69, 56.03, 60.42, 74.90, 128.30, 129.40, 135.77, 137.86; HRMS for $\mathrm{C}_{18} \mathrm{H}_{31} \mathrm{NO}_{2} \mathrm{SNa}\left(\mathrm{M}^{+}+\mathrm{Na}\right)$ : calcd. 348.1968, found: 348.1989 .

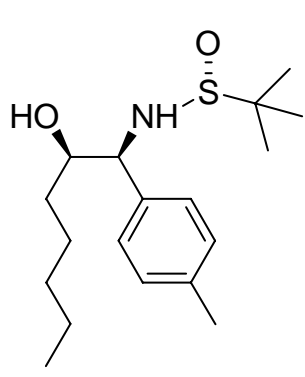

2d $[\alpha]_{\mathrm{D}}{ }^{20}=-30.2\left(c 0.30, \mathrm{CHCl}_{3}\right) ;{ }^{1} \mathrm{H} \mathrm{NMR}\left(300 \mathrm{MHz}, \mathrm{CDCl}_{3}\right): \delta$ $0.86(\mathrm{t}, 3 \mathrm{H}, J=6.9 \mathrm{~Hz}), 1.12-1.20(\mathrm{~m}, 2 \mathrm{H}), 1.28(\mathrm{~s}, 9 \mathrm{H})$, $1.29-1.36(\mathrm{~m}, 6 \mathrm{H}), 2.34(\mathrm{~s}, 3 \mathrm{H}), 3.78(\mathrm{~s}, 1 \mathrm{H}), 3.96(\mathrm{t}, 1 \mathrm{H}, J=4.5$ $\mathrm{Hz}), 4.35(\mathrm{dd}, 1 \mathrm{H}, J=3.6,5.4 \mathrm{~Hz}), 7.15(\mathrm{~d}, 1 \mathrm{H}, J=7.8 \mathrm{~Hz}), 7.23$ (d, 2H, $J=7.8 \mathrm{~Hz})$; FT-IR $\left(\mathrm{KBr}, \mathrm{cm}^{-1}\right): 3300,2959,2919,1462$, 1046; ESI-MS (m/z, \%): $326.2\left(\mathrm{M}^{+}+\mathrm{H}\right) ;{ }^{13} \mathrm{C} \mathrm{NMR}(75 \mathrm{MHz}$, $\left.\mathrm{CDCl}_{3}\right): \delta 13.90,21.00,22.46,22.58,25.51,31.64,33.29,56.13$, 62.17, 73.60, 128.11, 129.07, 135.23, 137.50; HRMS for $\mathrm{C}_{18} \mathrm{H}_{32} \mathrm{NO}_{2} \mathrm{~S}\left(\mathrm{M}^{+}+\mathrm{H}\right)$ : calcd. 326.2148, found: 326.2144 .

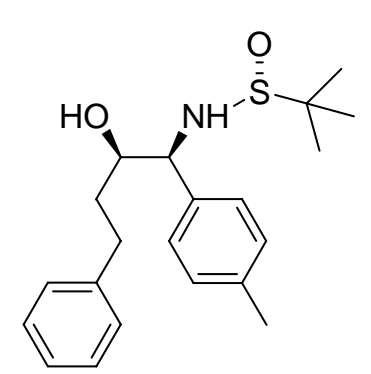

2e $[\alpha]_{\mathrm{D}}{ }^{20}=-53.8\left(\right.$ c $\left.1.35, \mathrm{CHCl}_{3}\right) ;{ }^{1} \mathrm{H} \mathrm{NMR}\left(300 \mathrm{MHz}, \mathrm{CDCl}_{3}\right)$ : $\delta 1.18(\mathrm{~s}, 1 \mathrm{H}), 1.22(\mathrm{~s}, 8 \mathrm{H}), 1.23-1.26(\mathrm{~m}, 1.4 \mathrm{H}), 1.48-1.50(\mathrm{~m}$, $1.4 \mathrm{H}), 2.33(\mathrm{~s}, 3 \mathrm{H}), 2.78-2.83(\mathrm{~m}, 2 \mathrm{H}), 2.64-2.67(\mathrm{~m}, 2 \mathrm{H}), 3.95$ (s, 1H), $3.99(\mathrm{~d}, 1 \mathrm{H}, J=6.3 \mathrm{~Hz}), 4.37(\mathrm{dd}, 1 \mathrm{H}, J=5.1,9.6 \mathrm{~Hz})$, 7.13-7.28 (m, 9H); FT-IR (KBr, $\mathrm{cm}^{-1}$ ): 3377, 3024, 2857, 1047, 1038; ESI-MS (m/z, \%): $360\left(\mathrm{M}^{+}+\mathrm{H}\right) ;{ }^{13} \mathrm{C}$ NMR $(75 \mathrm{MHz}$, $\left.\mathrm{CDCl}_{3}\right): \delta 21.01,22.62,32.17,35.10,56.21,62.26,73.02$, 125.76, 128.09, 128.22, 128.27, 128.33, 129.19, 135.10, 137.67, 141.70; HRMS for $\mathrm{C}_{21} \mathrm{H}_{30} \mathrm{NO}_{2} \mathrm{SNa}\left(\mathrm{M}^{+}+\mathrm{Na}\right)$ : calcd. 360.1992, found: 360.1998 .

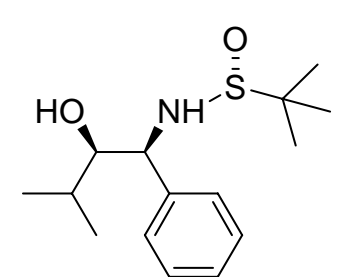

2f $[\alpha]_{\mathrm{D}}{ }^{20}=-51.3\left(c 0.65, \mathrm{CHCl}_{3}\right) ;{ }^{1} \mathrm{H} \mathrm{NMR}\left(300 \mathrm{MHz}, \mathrm{CDCl}_{3}\right): \delta$ $0.94(\mathrm{t}, 6 \mathrm{H}, J=6.9 \mathrm{~Hz}), 1.21(\mathrm{~s}, 9 \mathrm{H}), 1.48(\mathrm{~m}, 1 \mathrm{H}), 2.00(\mathrm{br}, 1 \mathrm{H})$, 3.63(br, $1 \mathrm{H}), 3.74(\mathrm{~d}, 1 \mathrm{H}, J=5.7 \mathrm{~Hz}), 4.49(\mathrm{dd}, 1 \mathrm{H}, J=5.7,4.5$ $\mathrm{Hz}), 7.31-7.43(\mathrm{~m}, 5 \mathrm{H})$; FT-IR $\left(\mathrm{KBr}, \mathrm{cm}^{-1}\right): 3410,3327,2956$, 1475, 1041, 699; ESI-MS (m/z, \%): $284.2\left(\mathrm{M}^{+}+\mathrm{H}\right) ;{ }^{13} \mathrm{C} \mathrm{NMR}$ $\left(75 \mathrm{MHz}, \mathrm{CDCl}_{3}\right): \delta 18.29,19.33,22.59,30.17,56.05,60.61,78.85,128.19,128.52$, 128.64, 138.40; HRMS for $\mathrm{C} 15 \mathrm{H} 25 \mathrm{NO} 2 \mathrm{SNa}\left(\mathrm{M}^{+}+\mathrm{Na}\right)$ : calcd.306.1498, found: 306.1515 .

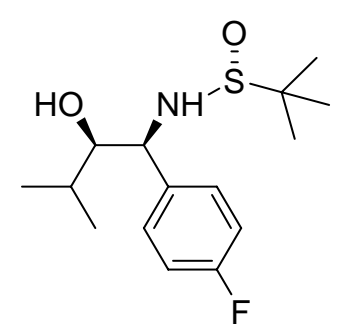

2g $[\alpha]_{\mathrm{D}}{ }^{20}=-42.1\left(\right.$ c 1.05, $\left.\mathrm{CHCl}_{3}\right) ;{ }^{1} \mathrm{H}$ NMR (300 MHz, $\left.\mathrm{CDCl}_{3}\right)$ : $\delta 0.94(\mathrm{~d}, 3 \mathrm{H}, J=1.5 \mathrm{~Hz}), 0.96(\mathrm{~d}, 3 \mathrm{H}, J=1.5 \mathrm{~Hz}), 1.22(\mathrm{~s}, 9 \mathrm{H})$, 1.24-1.27 (m, 1H), $2.12(\mathrm{~d}, 1 \mathrm{H}, J=2.1 \mathrm{~Hz}), 3.61(\mathrm{dd}, 1 \mathrm{H}, J=$ $3.9,7.8 \mathrm{~Hz}), 3.70(\mathrm{~d}, 1 \mathrm{H}, J=5.7 \mathrm{~Hz}), 4.48(\mathrm{dd}, 1 \mathrm{H}, J=5.7,9.3$ Hz), 7.05 (d, 2H, $J=18.1 \mathrm{~Hz}), 7.42$ (d, 2H, $J=18.1 \mathrm{~Hz}$ ); FT-IR $\left(\mathrm{KBr}, \mathrm{cm}^{-1}\right): 2961,2872,1604,1511,1048 ; \mathrm{ESI}-\mathrm{MS}(\mathrm{m} / \mathrm{z}, \%)$ : $302.2\left(\mathrm{M}^{+}+\mathrm{H}\right) ;{ }^{13} \mathrm{C}$ NMR $\left(75 \mathrm{MHz}, \mathrm{CDCl}_{3}\right): \delta 18.34,19.15,22.49,30.21,56.05$, $60.01,78.99,115.19,115.47,130.25,130.35,134.39,134.44,160.69,163.96$; HRMS for $\mathrm{C} 15 \mathrm{H} 25 \mathrm{NO} 2 \mathrm{SF}\left(\mathrm{M}^{+}+\mathrm{H}\right)$ : calcd. 302.1584, found: 302.1583 . 


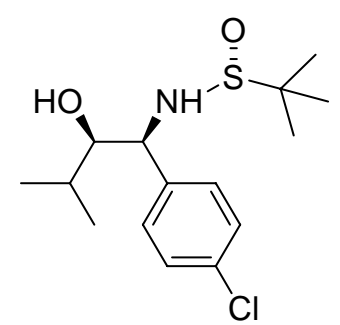

2h $[\alpha]_{\mathrm{D}}^{20}=-35.8\left(\right.$ c $\left.0.80, \mathrm{CHCl}_{3}\right) ;{ }^{1} \mathrm{H}$ NMR $\left(300 \mathrm{MHz}, \mathrm{CDCl}_{3}\right)$ : $\delta 0.94(\mathrm{~d}, 6 \mathrm{H}, J=6.6 \mathrm{~Hz}), 1.21(\mathrm{~s}, 9 \mathrm{H}), 1.45(\mathrm{~m}, 1 \mathrm{H}), 2.27$ (br, $1 \mathrm{H}), 3.60(\mathrm{~m}, 1 \mathrm{H}), 3.81(\mathrm{~m}, 1 \mathrm{H}), 4.44(\mathrm{~m}, 1 \mathrm{H}), 7.30(\mathrm{dd}, 2 \mathrm{H}, J=$ 2.4, $6.6 \mathrm{~Hz}), 7.36(\mathrm{dd}, 2 \mathrm{H}, J=2.4,6.6 \mathrm{~Hz})$; FT-IR $\left(\mathrm{KBr}, \mathrm{cm}^{-1}\right)$ : 3344, 2961, 1492, 1031; ESI-MS (m/z, \%): $318.2\left(\mathrm{M}^{+}+\mathrm{H}\right) ;{ }^{13} \mathrm{C}$ NMR $\left(75 \mathrm{MHz}, \mathrm{CDCl}_{3}\right): \delta 18.37,19.18,22.53,30.25,56.12$, 60.11, 78.99, 128.69, 130.03, 133.90, 137.04; HRMS for $\mathrm{C}_{15} \mathrm{H}_{24} \mathrm{NO}_{2} \mathrm{SClNa}\left(\mathrm{M}^{+}+\mathrm{Na}\right)$ : calcd. 340.1108 , found: 340.1113 .

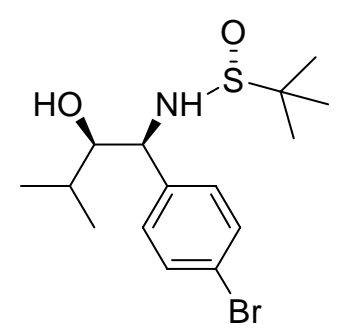

2i $[\alpha]_{\mathrm{D}}{ }^{20}=-26.7$ (c 0.75, $\left.\mathrm{CHCl}_{3}\right) ;{ }^{1} \mathrm{H} \mathrm{NMR}\left(300 \mathrm{MHz}, \mathrm{CDCl}_{3}\right): \delta$ $0.93(\mathrm{~d}, 6 \mathrm{H}, J=6.9 \mathrm{~Hz}), 1.20(\mathrm{~s}, 9 \mathrm{H}), 1.45(\mathrm{~m}, 1 \mathrm{H}), 2.35(\mathrm{~d}, 1 \mathrm{H}$, $J=5.1 \mathrm{~Hz}), 3.58(\mathrm{~m}, 1 \mathrm{H}), 3.84(\mathrm{~d}, 1 \mathrm{H}, J=6.6 \mathrm{~Hz}), 4.41(\mathrm{dd}, 1 \mathrm{H}$, $J=6.6,4.5 \mathrm{~Hz}), 7.30(\mathrm{~d}, 2 \mathrm{H}, J=8.4 \mathrm{~Hz}), 7.45(\mathrm{~d}, 2 \mathrm{H}, J=8.4$ Hz); FT-IR (KBr, $\left.\mathrm{cm}^{-1}\right): 3340,2958,1487,1031$; ESI-MS (m/z, $\%): 362.3\left(\mathrm{M}^{+}+\mathrm{H}\right) ;{ }^{13} \mathrm{C}$ NMR $\left(75 \mathrm{MHz}, \mathrm{CDCl}_{3}\right): \delta 18.37,19.18$, 22.52, 30.25, 56.13, 60.16, 78.98, 122.07, 130.36, 131.60, 137.61; HRMS for $\mathrm{C}_{15} \mathrm{H}_{24} \mathrm{NO}_{2} \mathrm{SBrNa}\left(\mathrm{M}^{+}+\mathrm{Na}\right)$ : calcd. 384.0603 , found: 384.0614 .

The X-ray structure is as follows:
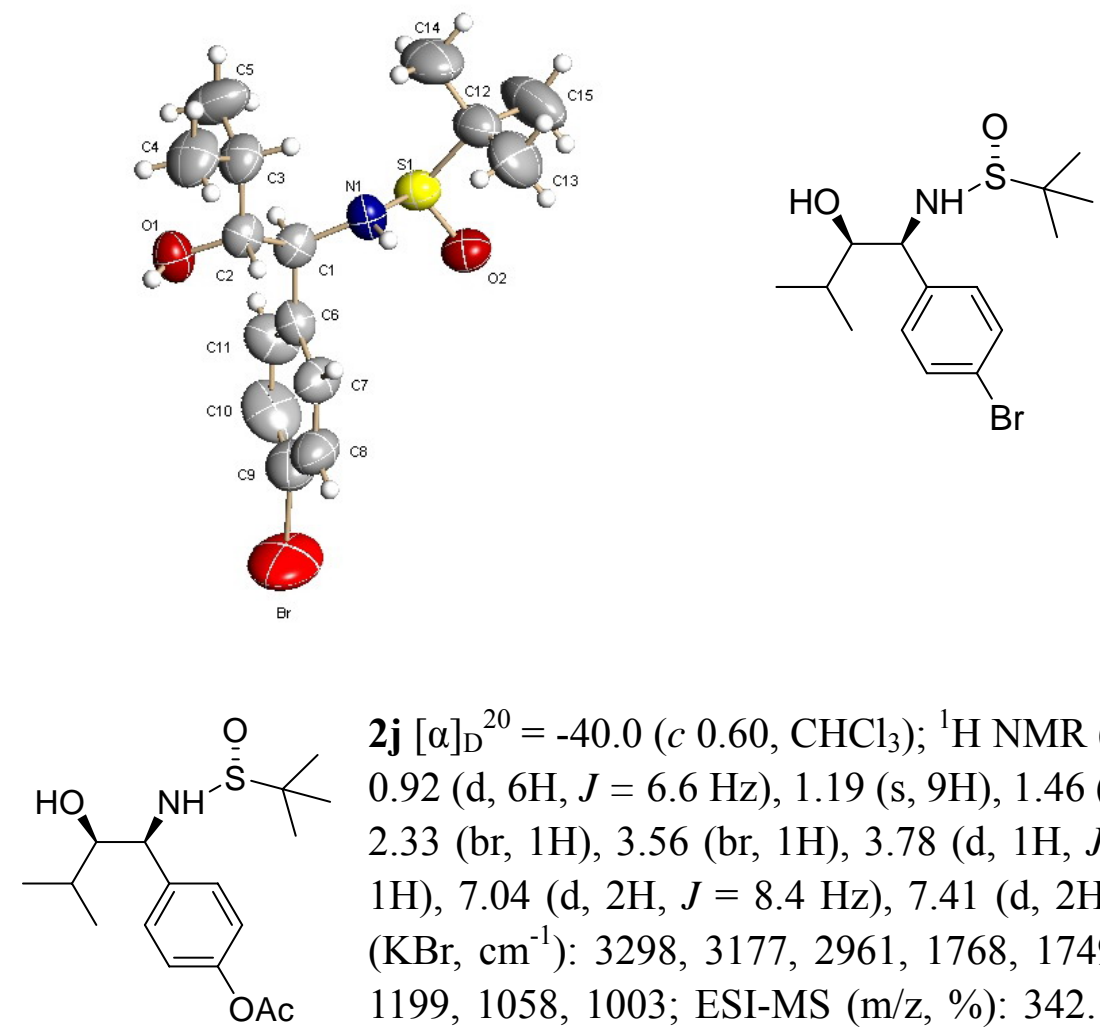

2j $[\alpha]_{\mathrm{D}}^{20}=-40.0\left(c 0.60, \mathrm{CHCl}_{3}\right) ;{ }^{1} \mathrm{H}$ NMR $\left(300 \mathrm{MHz}, \mathrm{CDCl}_{3}\right): \delta$ $0.92(\mathrm{~d}, 6 \mathrm{H}, J=6.6 \mathrm{~Hz}), 1.19(\mathrm{~s}, 9 \mathrm{H}), 1.46(\mathrm{~m}, 1 \mathrm{H}), 2.29(\mathrm{~s}, 3 \mathrm{H})$, $2.33(\mathrm{br}, 1 \mathrm{H}), 3.56(\mathrm{br}, 1 \mathrm{H}), 3.78(\mathrm{~d}, 1 \mathrm{H}, J=6.0 \mathrm{~Hz}), 4.45(\mathrm{~m}$, $1 \mathrm{H}), 7.04(\mathrm{~d}, 2 \mathrm{H}, J=8.4 \mathrm{~Hz}), 7.41(\mathrm{~d}, 2 \mathrm{H}, J=8.4 \mathrm{~Hz})$; FT-IR $\left(\mathrm{KBr}, \mathrm{cm}^{-1}\right): 3298,3177,2961,1768,1749,1507,1370,1222$, 1199, 1058, 1003; ESI-MS (m/z, \%): $342.1\left(\mathrm{M}^{+}+\mathrm{H}\right) ;{ }^{13} \mathrm{C}$ NMR $\left(75 \mathrm{MHz}, \mathrm{CDCl}_{3}\right): \delta 18.29,19.23,21.09,22.53,30.07,56.03,60.03,78.77,121.52$, 129.69, 136.07, 150.23, 169.36; HRMS for $\mathrm{C}_{17} \mathrm{H}_{27} \mathrm{NO}_{4} \mathrm{SNa}\left(\mathrm{M}^{+}+\mathrm{Na}\right)$ : calcd. 364.1553, found: 364.1547 . 


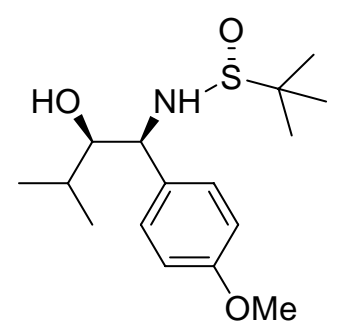

2k $[\alpha]_{\mathrm{D}}{ }^{20}=-38.1\left(\right.$ c $\left.1.55, \mathrm{CHCl}_{3}\right) ;{ }^{1} \mathrm{H}$ NMR $\left(300 \mathrm{MHz}, \mathrm{CDCl}_{3}\right)$ : $\delta 0.94(\mathrm{~s}, 3 \mathrm{H}), 0.97(\mathrm{~s}, 3 \mathrm{H}),, 1.22(\mathrm{~s}, 9 \mathrm{H}), 1.25-1.29(\mathrm{~m}, 1 \mathrm{H})$, $2.06(\mathrm{~d}, 1 \mathrm{H}, J=4.2 \mathrm{~Hz}), 3.63(\mathrm{~m}, 2 \mathrm{H}), 3.80(\mathrm{~s}, 3 \mathrm{H}), 4.45(\mathrm{t}, 1 \mathrm{H}$, $J=4.8 \mathrm{~Hz}), 6.89(\mathrm{~d}, 2 \mathrm{H}, J=8.7 \mathrm{~Hz}), 7.34(\mathrm{~d}, 2 \mathrm{H}, J=8.7 \mathrm{~Hz})$; FT-IR $\left(\mathrm{KBr}, \mathrm{cm}^{-1}\right)$ : 3504, 3139, 2962, 1515, 1247, 1039, 1001; ESI-MS (m/z, \%): $314\left(\mathrm{M}^{+}+\mathrm{H}\right) ;{ }^{13} \mathrm{C}$ NMR $\left(75 \mathrm{MHz}, \mathrm{CDCl}_{3}\right): \delta$ $18.23,19.20,22.49,30.13,55.07,55.91,60.19,78.87,113.81,129.65,130.51,159.15$; HRMS for $\mathrm{C}_{16} \mathrm{H}_{27} \mathrm{NO}_{3} \mathrm{SNa}\left(\mathrm{M}^{+}+\mathrm{Na}\right)$ : calcd. 336.1609, found: 336.1607 .

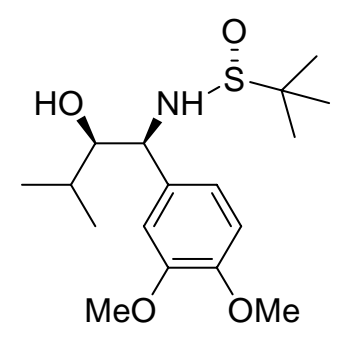

$2 \mathbf{[}[\alpha]_{\mathrm{D}}{ }^{20}=-35.2\left(c 0.55, \mathrm{CHCl}_{3}\right) ;{ }^{1} \mathrm{H} \mathrm{NMR}\left(300 \mathrm{MHz}, \mathrm{CDCl}_{3}\right): \delta$ $0.96(\mathrm{~s}, 3 \mathrm{H}), 0.99(\mathrm{~s}, 3 \mathrm{H}),$,1.24 (s, 9H), $2.01(\mathrm{~d}, 1 \mathrm{H}, J=4.2 \mathrm{~Hz})$, 3.59-3.64 (m, 2H), $3.90(\mathrm{~s}, 3 \mathrm{H}), 4.43(\mathrm{dd}, 1 \mathrm{H}, J=5.1,9.6 \mathrm{~Hz})$, $6.86(\mathrm{dd}, 1 \mathrm{H}, J=6.0,8.7 \mathrm{~Hz}), 6.99(\mathrm{dd}, 2 \mathrm{H}, J=4.8,8.7 \mathrm{~Hz})$; FT-IR $\left(\mathrm{KBr}, \mathrm{cm}^{-1}\right): 3428,2960,2837,1522,1041,1025$; ESI-MS $(\mathrm{m} / \mathrm{z}, \%): 344\left(\mathrm{M}^{+}+\mathrm{H}\right) ;{ }^{13} \mathrm{C}$ NMR $\left(75 \mathrm{MHz}, \mathrm{CDCl}_{3}\right): \delta 18.07$, 19.29, 22.46, 30.06, 55.66, 55.80, 55.88, 60.52, 78.77, 110.93, 111.79, 120.71, 130.98, 148.69; HRMS for $\mathrm{C}_{17} \mathrm{H}_{29} \mathrm{NO}_{4} \mathrm{SNa}\left(\mathrm{M}^{+}+\mathrm{Na}\right)$ : calcd. 366.1709, found: 366.1712 .

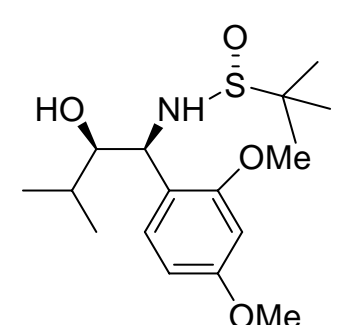

$2 \mathbf{m}[\alpha]_{\mathrm{D}}{ }^{20}=-49.6\left(\right.$ c $\left.0.70, \mathrm{CHCl}_{3}\right) ;{ }^{1} \mathrm{H}$ NMR $\left(300 \mathrm{MHz}, \mathrm{CDCl}_{3}\right)$ : $\delta 0.93(\mathrm{~d}, 3 \mathrm{H}, J=6.6 \mathrm{~Hz}), 0.97(\mathrm{~d}, 3 \mathrm{H}, J=6.6 \mathrm{~Hz}), 1.22(\mathrm{~s}, 9 \mathrm{H})$, $1.73-1.80(\mathrm{~m}, 1 \mathrm{H}), 1.94(\mathrm{~s}, 1 \mathrm{H}), 3.68(\mathrm{~s}, 1 \mathrm{H}), 3.79(\mathrm{~s}, 3 \mathrm{H}), 3.84$ (s, $3 \mathrm{H}), 4.13(\mathrm{dd}, 1 \mathrm{H}, J=7.5,16 \mathrm{~Hz}), 4.47(\mathrm{dd}, 1 \mathrm{H}, J=7.5,15$ $\mathrm{Hz}), 6.46-6.49(\mathrm{~m}, 2 \mathrm{H}), 7.27(\mathrm{dd}, 1 \mathrm{H}, J=3.6,8.7 \mathrm{~Hz})$; FT-IR $\left(\mathrm{KBr}, \mathrm{cm}^{-1}\right): 3541,2964,1612,1071$; ESI-MS (m/z, \%): 344.2 $\left(\mathrm{M}^{+}+\mathrm{H}\right) ;{ }^{13} \mathrm{C}$ NMR $\left(75 \mathrm{MHz}, \mathrm{CDCl}_{3}\right): \delta 16.31,19.74,22.49,29.67,55.21,55.33$, $55.77,59.17,78.10,99.12,104.47,119.77,130.95,157.93,160.48$; HRMS for $\mathrm{C}_{17} \mathrm{H}_{29} \mathrm{NO}_{4} \mathrm{SNa}\left(\mathrm{M}^{+}+\mathrm{Na}\right)$ : calcd. 366.1709, found: 366.1706 .

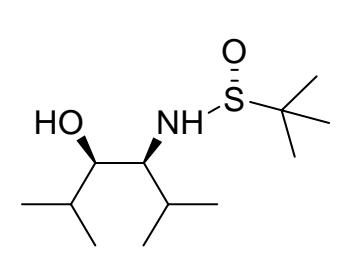

2n $[\alpha]_{\mathrm{D}}{ }^{20}=-62.8\left(\right.$ c $\left.0.50, \mathrm{CHCl}_{3}\right) ;{ }^{1} \mathrm{H}$ NMR $\left(300 \mathrm{MHz}, \mathrm{CDCl}_{3}\right)$ : $\delta$ 0.91-0.97 (m, 9H), $1.09(\mathrm{~d}, 3 \mathrm{H}, J=5.1 \mathrm{~Hz}), 1.26(\mathrm{~s}, 9 \mathrm{H}), 1.66$ $(\mathrm{d}, 1 \mathrm{H}, J=4.8 \mathrm{~Hz}), 1.91-1.95(\mathrm{~m}, 1 \mathrm{H}), 2.20-2.25(\mathrm{~m}, 1 \mathrm{H})$, 3.16-3.20 (m, 1H), $3.23(\mathrm{~d}, 1 \mathrm{H}, J=6.6, \mathrm{~Hz}), 3.34(\mathrm{~d}, 1 \mathrm{H}, J=$ 6.0Hz); FT-IR $\left(\mathrm{KBr}, \mathrm{cm}^{-1}\right): 3367,2964,2874,1473,1035$; ESI-MS (m/z, \%): $250.2\left(\mathrm{M}^{+}+\mathrm{H}\right) ;{ }^{13} \mathrm{C} \mathrm{NMR}\left(75 \mathrm{MHz}, \mathrm{CDCl}_{3}\right): \delta 16.62,16.79,19.81$, $20.87,22.70,27.25,29.28,56.10,62.70,78.15$; HRMS for $\mathrm{C} 12 \mathrm{H} 27 \mathrm{NO} 2 \mathrm{SNa}$ $\left(\mathrm{M}^{+}+\mathrm{Na}\right)$ : calcd. 272.1654, found: 272.1657 .

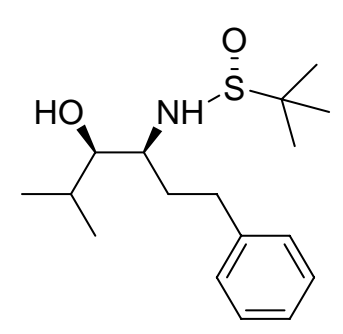

2o $[\alpha]_{\mathrm{D}}{ }^{20}=-118.8\left(\right.$ c $\left.0.30, \mathrm{CHCl}_{3}\right) ;{ }^{1} \mathrm{H}$ NMR $\left(300 \mathrm{MHz}, \mathrm{CDCl}_{3}\right)$ : $\delta 0.68(\mathrm{~d}, 3 \mathrm{H}, J=6.9 \mathrm{~Hz}), 0.98(\mathrm{~d}, 3 \mathrm{H}, J=6.9 \mathrm{~Hz}), 1.26(\mathrm{~s}, 9 \mathrm{H})$, $1.66-1.68(\mathrm{~m}, 1 \mathrm{H}), 1.91-2.05(\mathrm{~m}, 2 \mathrm{H}), 2.64-2.69(\mathrm{~m}, 1 \mathrm{H})$, 2.87-2.89 (m, 1H), $3.20(\mathrm{dd}, 2 \mathrm{H}, J=2.1,9.0 \mathrm{~Hz}), 3.31-3.38(\mathrm{~m}$, 2H), 7.18-7.32 (m, 5H); FT-IR (KBr, $\left.\mathrm{cm}^{-1}\right)$ : 3370, 2959, 5870, 1042; ESI-MS (m/z, \%): $312.2\left(\mathrm{M}^{+}+\mathrm{H}\right) ;{ }^{13} \mathrm{C}$ NMR $(75 \mathrm{MHz}$, 
$\left.\mathrm{CDCl}_{3}\right): \delta 18.64,19.69,22.72,28.55,30.25,31.66,55.68,58.09,80.35,125.90$, 128.35, 128.55, 141.51; $\mathrm{HRMS} \quad \mathrm{C}_{17} \mathrm{H}_{29} \mathrm{NO}_{2} \mathrm{SNa} \quad\left(\mathrm{M}^{+}+\mathrm{Na}\right): \quad$ calcd.334.1811, found $: 334.1813$.

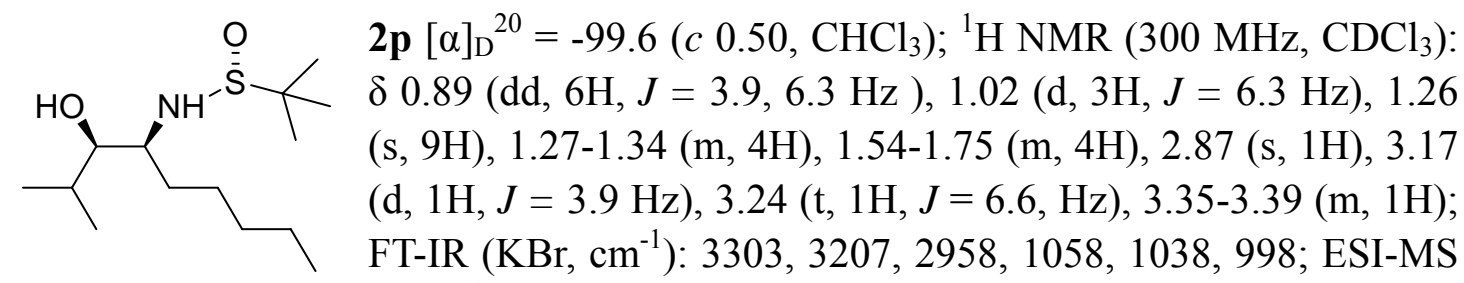
$(\mathrm{m} / \mathrm{z}, \%): 278.2\left(\mathrm{M}^{+}+\mathrm{H}\right) ;{ }^{13} \mathrm{C} \mathrm{NMR}\left(75 \mathrm{MHz}, \mathrm{CDCl}_{3}\right): \delta 14.00,19.02,19.58,22.46$, 22.64, 25.49, 26.88, 30.13, 31.60, 55.59, 58.74, 79.99; HRMS for $\mathrm{C}_{14} \mathrm{H}_{31} \mathrm{NO}_{2} \mathrm{SNa}$ $\left(\mathrm{M}^{+}+\mathrm{Na}\right)$ : calcd. 300.1968 , found: 300.1970 .<smiles>CC(C)[C@H](O)[C@H](COCc1ccccc1)N[SH](=O)C(C)(C)C</smiles>
FT-IR (film, cm ${ }^{-1}$ ): 3400 (br), 2960, 1072, 1051; ESI-MS (m/z, \%): $328.3\left(\mathrm{M}^{+}+\mathrm{H}\right)$; HRMS for $\mathrm{C}_{17} \mathrm{H}_{29} \mathrm{NO}_{3} \mathrm{SNa}\left(\mathrm{M}^{+}+\mathrm{Na}\right)$ : calcd. 350.1760 , found: 350.1764 .

\section{Determination of the diastereoselectivity and the enantiomeric excess.}

The diastereoselectivity of the reaction was measured according to HPLC-MS and ${ }^{1} \mathrm{H}$ NMR of the crude products. The major diastereomer were then separated by column chromatography.

The optical purities of the obtained $\beta$-amino alcohol products were determined by measuring the ee values of their diacetate or benzoylate derivatives.<smiles>[R2]C(O)C([R1])NS(C)(C)C</smiles>

2<smiles>[R2]C(O)C([R1])NS(C)(C)C</smiles>

$2 n, 2 p$ $\frac{\text { 1. } \mathrm{HCl} / \mathrm{MeOH}, \mathrm{rt}}{\text { 2. } \mathrm{Ac}_{2} \mathrm{O}, \mathrm{Et}_{3} \mathrm{~N}, \mathrm{DMAP},}$ $\underset{\text { 1. } \mathrm{HCl} / \mathrm{MeOH}, \mathrm{rt}}{\stackrel{\substack{\mathrm{Bz}_{2} \mathrm{O}, \mathrm{Et}_{3} \mathrm{~N}, \mathrm{DMAP} \\ \mathrm{CH}_{2} \mathrm{Cl}_{2}, \mathrm{rt}}}{\longrightarrow}}$
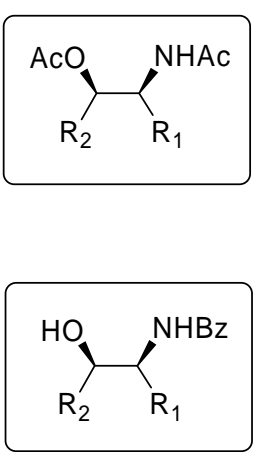


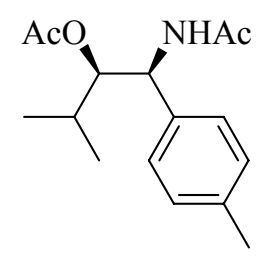

HPLC: Chiralpak OD column; detected at $214 \mathrm{~nm}$, eluent: $n$-hexane/iso-propanol $=95 / 5(\mathrm{v} / \mathrm{v})$.

$[\alpha]_{\mathrm{D}}{ }^{20}=+153.6\left(\right.$ c $\left.0.17, \mathrm{CHCl}_{3}\right) ;{ }^{1} \mathrm{H}$ NMR $\left(300 \mathrm{MHz}, \mathrm{CDCl}_{3}\right): \delta 0.91$ $(\mathrm{d}, 3 \mathrm{H}, J=6.6 \mathrm{~Hz}), 0.99(\mathrm{~d}, 3 \mathrm{H}, J=6.6 \mathrm{~Hz}), 1.77-1.83(\mathrm{~m}, 1 \mathrm{H})$, $1.91(\mathrm{~s}, 3 \mathrm{H}), 1.94(\mathrm{~s}, 3 \mathrm{H}), 2.32(\mathrm{~s}, 3 \mathrm{H}), 4.99(\mathrm{dd}, 1 \mathrm{H}, J=6.6,12.6$ $\mathrm{Hz}), 5.22(\mathrm{dd}, 1 \mathrm{H}, J=5.4,8.7 \mathrm{~Hz}), 6.39(\mathrm{~d}, 1 \mathrm{H}, J=8.7 \mathrm{~Hz}), 7.12(\mathrm{~d}, 2 \mathrm{H}, J=8.1 \mathrm{~Hz})$, 7.19 (d, 2H, $J=8.1 \mathrm{~Hz})$; FT-IR $\left(\mathrm{KBr}, \mathrm{cm}^{-1}\right): 3282,2966,1745,1655,1235$; ESI-MS $(\mathrm{m} / \mathrm{z}, \%): 278\left(\mathrm{M}^{+}+\mathrm{H}\right) ;{ }^{13} \mathrm{C}$ NMR $\left(75 \mathrm{MHz}, \mathrm{CDCl}_{3}\right): \delta 17.59,19.47,20.77,21.05$, 23.29, 29.24, 53.80, 79.88, 127.72, 129.02, 135.35, 137.33, 169.08, 171.14; HRMS for $\mathrm{C}_{16} \mathrm{H}_{23} \mathrm{NO}_{3} \mathrm{Na}\left(\mathrm{M}^{+}+\mathrm{Na}\right)$ : calcd. 300.1570 , found: 300.1573 .

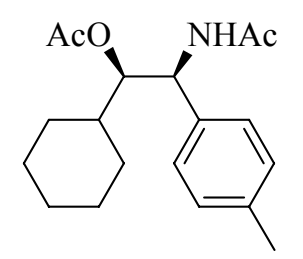

HPLC: Chiralcel OD column; detected at $214 \mathrm{~nm}$, eluent: $n$-hexane/iso-propanol $=95 / 5(\mathrm{v} / \mathrm{v})$.

$[\alpha]_{\mathrm{D}}{ }^{20}=+116.3\left(\right.$ c $\left.0.13, \mathrm{CHCl}_{3}\right) ;{ }^{1} \mathrm{H}$ NMR $\left(300 \mathrm{MHz}, \mathrm{CDCl}_{3}\right): \delta$ $1.00-1.78(\mathrm{~m}, 11 \mathrm{H}), 1.89(\mathrm{~d}, 3 \mathrm{H}, J=3.0 \mathrm{~Hz}), 1.93(\mathrm{~d}, 3 \mathrm{H}, J=3.0$ $\mathrm{Hz}), 2.27(\mathrm{~s}, 3 \mathrm{H}), 4.95(\mathrm{dd}, 1 \mathrm{H}, J=4.8,7.2 \mathrm{~Hz}), 5.20(\mathrm{dd}, 1 \mathrm{H}, J=$ 4.8, $8.4 \mathrm{~Hz}), 6.35$ (s, 1H), 7.07 (d, 2H, $J=8.1 \mathrm{~Hz}), 7.13$ (d, 2H, $J=8.1 \mathrm{~Hz})$; FT-IR $\left(\mathrm{KBr}, \mathrm{cm}^{-1}\right): 3293,2933,2854,1743,1731,1654,1234 ;$ ESI-MS (m/z, \%): 318 $\left(\mathrm{M}^{+}+\mathrm{H}\right) ;{ }^{13} \mathrm{C} \mathrm{NMR}\left(75 \mathrm{MHz}, \mathrm{CDCl}_{3}\right): \delta 20.80,21.05,23.29,25.55,25.71,26.06$, 27.87, 29.65, 38.64, 53.26, 79.41, 127.75, 129.02; HRMS for $\mathrm{C}_{19} \mathrm{H}_{28} \mathrm{NO}_{3}\left(\mathrm{M}^{+}+\mathrm{H}\right)$ : calcd. 318.2064 , found: 318.2063 .

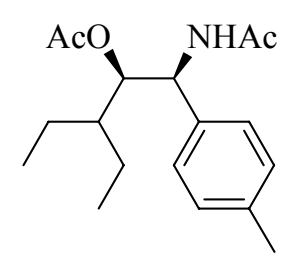

HPLC: Chiralpak AD-H column; detected at $254 \mathrm{~nm}$, eluent: $n$-hexane/iso-propanol $=80 / 20(\mathrm{v} / \mathrm{v})$.

M.p.: $105^{\circ} \mathrm{C} ;[\alpha]_{\mathrm{D}}{ }^{20}=+127.2\left(c 0.24, \mathrm{CHCl}_{3}\right) ;{ }^{1} \mathrm{H} \mathrm{NMR}(300 \mathrm{MHz}$, $\left.\mathrm{CDCl}_{3}\right): \delta$ 0.81-1.22 (m, 6H), 1.43-1.55 (m, 5H), $1.93(\mathrm{~s}, 3 \mathrm{H}), 1.96$ $(\mathrm{s}, 3 \mathrm{H}), 2.34(\mathrm{~s}, 3 \mathrm{H}), 5.17(\mathrm{dd}, 1 \mathrm{H}, J=5.7,8.1 \mathrm{~Hz}), 5.26(\mathrm{dd}, 1 \mathrm{H}, J$ $=5.7,8.1 \mathrm{~Hz}), 6.16(\mathrm{~d}, 1 \mathrm{H}, J=7.2 \mathrm{~Hz}), 7.13(\mathrm{~d}, 2 \mathrm{H}, J=8.1 \mathrm{~Hz}), 7.18(\mathrm{~d}, 2 \mathrm{H}, J=8.1$ Hz); FT-IR (KBr, cm $\left.{ }^{-1}\right): 3271,2964,1737,1651,1519,1240$; ESI-MS (m/z, \%): 306 $\left(\mathrm{M}^{+}+\mathrm{H}\right) ;{ }^{13} \mathrm{C} \mathrm{NMR}\left(75 \mathrm{MHz}, \mathrm{CDCl}_{3}\right): \delta 10.58,10.91,20.63,20.74,21.01,21.64$, 23.20, 53.67, 77.42, 127.73, 128.91, 135.50, 137.15, 169.05, 170.95; HRMS for $\mathrm{C}_{18} \mathrm{H}_{27} \mathrm{NO}_{3} \mathrm{Na}\left(\mathrm{M}^{+}+\mathrm{Na}\right)$ : calcd. 328.1883 , found: 328.1884 .

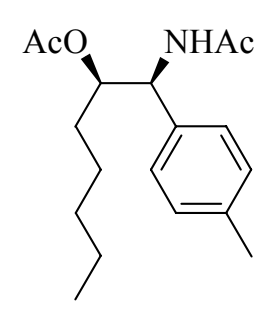

HPLC: Chiralpak AD-H column; detected at $214 \mathrm{~nm}$, eluent: $n$-hexane/iso-propanol $=90 / 10(\mathrm{v} / \mathrm{v})$.

$[\alpha]_{\mathrm{D}}{ }^{20}=+83\left(\right.$ c $\left.0.27, \mathrm{CHCl}_{3}\right) ;{ }^{1} \mathrm{H}$ NMR $\left(300 \mathrm{MHz}, \mathrm{CDCl}_{3}\right): \delta 0.85(\mathrm{t}$, $3 \mathrm{H}, J=6.9 \mathrm{~Hz}), 1.23-1.49(\mathrm{~m}, 8 \mathrm{H}), 1.97(\mathrm{~s}, 3 \mathrm{H}), 2.00(\mathrm{~s}, 3 \mathrm{H})$, 1.77-1.83 (m, 1H), $2.33(\mathrm{~s}, 3 \mathrm{H}), 5.14(\mathrm{dd}, 2 \mathrm{H}, J=7.2,12 \mathrm{~Hz}), 6.52$ $(\mathrm{d}, 1 \mathrm{H}, J=6.6 \mathrm{~Hz}), 7.12(\mathrm{~d}, 2 \mathrm{H}, J=8.1 \mathrm{~Hz}), 7.17(\mathrm{~d}, 2 \mathrm{H}, J=8.1$ $\mathrm{Hz})$; FT-IR (KBr, cm $\left.{ }^{-1}\right)$ : 3244, 2960, 1736, 1648, 1244; ESI-MS (m/z, \%): 306 $\left(\mathrm{M}^{+}+\mathrm{H}\right) ;{ }^{13} \mathrm{C} \mathrm{NMR}\left(75 \mathrm{MHz}, \mathrm{CDCl}_{3}\right): \delta 13.90,21.01,21.04,22.40,23.32,25.16$, 31.00, 31.40, 55.63, 77.43, 127.48, 129.03, 134.87, 137.28, 169.23, 171.26; HRMS for $\mathrm{C}_{18} \mathrm{H}_{27} \mathrm{NO}_{3} \mathrm{Na}\left(\mathrm{M}^{+}+\mathrm{Na}\right)$ : calcd. 328.1883 , found: 328.1886 . 


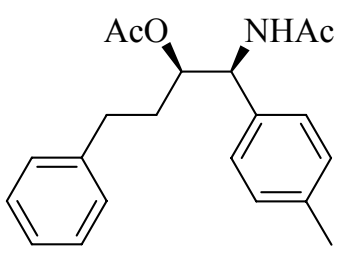

HPLC: Chiralpak AD column; detected at $254 \mathrm{~nm}$, eluent: $n$-hexane/iso-propanol $=80 / 20(\mathrm{v} / \mathrm{v})$.

$[\alpha]_{\mathrm{D}}^{20}=+69.5\left(c \quad 0.15, \mathrm{CHCl}_{3}\right) ;{ }^{1} \mathrm{H} \mathrm{NMR}\left(300 \mathrm{MHz}, \mathrm{CDCl}_{3}\right): \delta$ $1.79-1.84(\mathrm{~m}, 2 \mathrm{H}), 1.95(\mathrm{~d}, 3 \mathrm{H}, J=4.5 \mathrm{~Hz}), 2.00(\mathrm{~d}, 3 \mathrm{H}, J=$ $4.5 \mathrm{~Hz}), 2.31(\mathrm{~s}, 3 \mathrm{H}), 2.56-2.66(\mathrm{~m}, 2 \mathrm{H}), 5.16(\mathrm{dd}, 1 \mathrm{H}, J=4.5$, $12 \mathrm{~Hz}), 6.46(\mathrm{~d}, 1 \mathrm{H}, J=7.8 \mathrm{~Hz}), 7.09-7.28(\mathrm{~m}, 5 \mathrm{H})$; FT-IR $\left(\mathrm{KBr}, \mathrm{cm}^{-1}\right): 3356,2955$, 1731, 1653, 1244; ESI-MS (m/z, \%): $340\left(\mathrm{M}^{+}+\mathrm{H}\right) ;{ }^{13} \mathrm{C}$ NMR $\left(75 \mathrm{MHz}, \mathrm{CDCl}_{3}\right): \delta$ 20.96, 21.04, 23.31, 31.84, 32.84, 55.69, 75.48, 125.99, 127.41, 128.27, 128.39, 129.11, 134.64, 137.43, 140.91, 169.32, 171.28; HRMS for $\mathrm{C}_{21} \mathrm{H}_{25} \mathrm{NO}_{3} \mathrm{Na}\left(\mathrm{M}^{+}+\mathrm{Na}\right)$ : calcd. 362.1727 , found: 362.1722 .

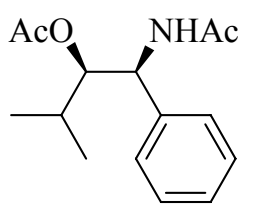

HPLC: Chiralpak AD-H column; detected at $254 \mathrm{~nm}$, eluent: $n$-hexane/iso-propanol $=80 / 20(\mathrm{v} / \mathrm{v})$.

$[\alpha]_{\mathrm{D}}{ }^{20}=+164.7$ (c 0.18, $\left.\mathrm{CHCl}_{3}\right) ;{ }^{1} \mathrm{H} \mathrm{NMR}\left(300 \mathrm{MHz}, \mathrm{CDCl}_{3}\right): \delta 0.92$ $(\mathrm{d}, 3 \mathrm{H}, J=6.6 \mathrm{~Hz}), 0.97(\mathrm{~d}, 3 \mathrm{H}, J=6.6 \mathrm{~Hz}), 1.81-1.87(\mathrm{~m}, 1 \mathrm{H})$, 1.92 (s, 6H), $5.01(\mathrm{t}, 1 \mathrm{H}, J=6.3 \mathrm{~Hz}), 5.25(\mathrm{dd}, 1 \mathrm{H}, J=6.0,8.7 \mathrm{~Hz}), 6.68(\mathrm{~s}, 1 \mathrm{H}), 7.27$ (m, 5H); FT-IR $\left(\mathrm{KBr}, \mathrm{cm}^{-1}\right): 3645,3314,1730,1650,1548,1247$; ESI-MS (m/z, \%): $264\left(\mathrm{M}^{+}+\mathrm{H}\right) ;{ }^{13} \mathrm{C}$ NMR $\left(75 \mathrm{MHz}, \mathrm{CDCl}_{3}\right): \delta 17.48,19.50,20.72,23.29$ 29.21, 54.06, $79.75,127.68,127.81,128.32,138.42,169.18,171.14$; HRMS for $\mathrm{C}_{15} \mathrm{H}_{22} \mathrm{NO}_{3}$ $\left(\mathrm{M}^{+}+\mathrm{H}\right)$ : calcd. 264.1597, found: 264.1597 .

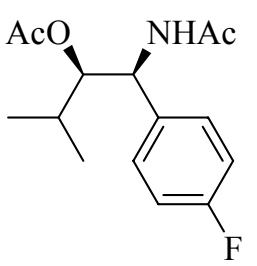

HPLC: Chiralcel OD column; detected at $214 \mathrm{~nm}$, eluent: $n$-hexane/iso-propanol $=95 / 5(\mathrm{v} / \mathrm{v})$.

$[\alpha]_{\mathrm{D}}^{20}=+123.1\left(c 0.11, \mathrm{CHCl}_{3}\right) ;{ }^{1} \mathrm{H} \mathrm{NMR}\left(300 \mathrm{MHz}, \mathrm{CDCl}_{3}\right): \delta 0.91$ $(\mathrm{d}, 3 \mathrm{H}, J=6.9 \mathrm{~Hz}), 1.00(\mathrm{~d}, 3 \mathrm{H}, J=6.9 \mathrm{~Hz}), 1.75-1.82(\mathrm{~m}, 1 \mathrm{H})$, $1.97(\mathrm{~s}, 3 \mathrm{H}), 1.98(\mathrm{~s}, 3 \mathrm{H}), 4.94(\mathrm{dd}, 1 \mathrm{H}, J=4.8,7.2 \mathrm{~Hz}), 5.24(\mathrm{dd}, 1 \mathrm{H}$, $J=5.4,8.4 \mathrm{~Hz}), 6.21(\mathrm{~d}, 1 \mathrm{H}, J=7.8 \mathrm{~Hz}), 7.01(\mathrm{~d}, 2 \mathrm{H}, J=8.1 \mathrm{~Hz}), 7.26(\mathrm{~d}, 2 \mathrm{H}, J=$ $8.1 \mathrm{~Hz})$; FT-IR (KBr, $\left.\mathrm{cm}^{-1}\right): 3277,2968,1743,1513,1232$; ESI-MS (m/z, \%): 281 $\left(\mathrm{M}^{+}+\mathrm{H}\right) ;{ }^{13} \mathrm{C}$ NMR $\left(75 \mathrm{MHz}, \mathrm{CDCl}_{3}\right): \delta 17.11,19.42,20.65,23.12,29.10,53.37$, 79.32, 115.01, 129.45, 134.55, 162.00, 169.33, 170.99; HRMS for $\mathrm{C}_{15} \mathrm{H}_{20} \mathrm{NO}_{3} \mathrm{NaF}$ $\left(\mathrm{M}^{+}+\mathrm{Na}\right)$ : calcd. 300.1570 , found: 304.1321 .

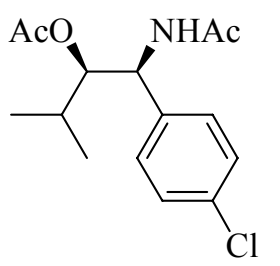

HPLC: Chiralpak AD column; detected at $214 \mathrm{~nm}$, eluent: $n$-hexane/iso-propanol $=80 / 20(\mathrm{v} / \mathrm{v})$.

$[\alpha]_{\mathrm{D}}^{20}=+153.7\left(\right.$ c $\left.0.15, \mathrm{CHCl}_{3}\right) ;{ }^{1} \mathrm{H}$ NMR $\left(300 \mathrm{MHz}, \mathrm{CDCl}_{3}\right): \delta$ $0.91(\mathrm{~d}, 3 \mathrm{H}, J=6.6 \mathrm{~Hz}), 0.98(\mathrm{~d}, 3 \mathrm{H}, J=6.6 \mathrm{~Hz}), 1.78-1.84(\mathrm{~m}$, $1 \mathrm{H}), 1.92(\mathrm{~s}, 3 \mathrm{H}), 1.96(\mathrm{~s}, 3 \mathrm{H}), 4.95(\mathrm{t}, 1 \mathrm{H}, J=6.9 \mathrm{~Hz}), 5.21(\mathrm{dd}, 1 \mathrm{H}$, $J=5.7,8.4 \mathrm{~Hz}), 6.62(\mathrm{~d}, 1 \mathrm{H}, J=8.1 \mathrm{~Hz}), 7.23(\mathrm{~d}, 2 \mathrm{H}, J=8.1 \mathrm{~Hz}), 7.28(\mathrm{~d}, 2 \mathrm{H}, J=$ $8.1 \mathrm{~Hz})$; FT-IR (KBr, cm $\left.{ }^{-1}\right): 3302,2976,1737,1646,1542,1236$; ESI-MS (m/z, \%): 298, $300\left(\mathrm{M}^{+}+\mathrm{H}\right) ;{ }^{13} \mathrm{C}$ NMR $\left(75 \mathrm{MHz}, \mathrm{CDCl}_{3}\right): \delta 17.49,19.46,20.75,23.27,29.22$, 53.06, 79.65, 128.46, 129.17, 133.44, 137.04, 169.19, 171.21; HRMS for $\mathrm{C}_{15} \mathrm{H}_{20} \mathrm{NO}_{3} \mathrm{NaCl}\left(\mathrm{M}^{+}+\mathrm{Na}\right)$ : calcd. 320.1024 , found: 320.1024 . 


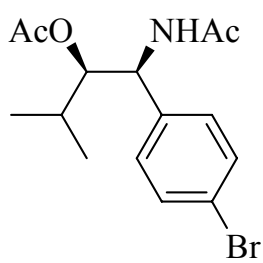

HPLC: Chiralpak AD column; detected at $254 \mathrm{~nm}$, eluent: $n$-hexane/iso-propanol $=80 / 20(\mathrm{v} / \mathrm{v})$.

$[\alpha]_{\mathrm{D}}{ }^{20}=+155.6\left(\right.$ c $\left.0.11, \mathrm{CHCl}_{3}\right) ;{ }^{1} \mathrm{H} \mathrm{NMR}\left(300 \mathrm{MHz}, \mathrm{CDCl}_{3}\right): \delta$ $0.91(\mathrm{~d}, 3 \mathrm{H}, J=6.6 \mathrm{~Hz}), 0.97(\mathrm{~d}, 3 \mathrm{H}, J=6.6 \mathrm{~Hz}), 1.78-1.84(\mathrm{~m}$, $1 \mathrm{H}), 1.92(\mathrm{~s}, 3 \mathrm{H}), 1.95(\mathrm{~s}, 3 \mathrm{H}), 4.95(\mathrm{dd}, 1 \mathrm{H}, J=5.7,6.6 \mathrm{~Hz}), 5.19$ $(\mathrm{dd}, 1 \mathrm{H}, J=6.3,8.1 \mathrm{~Hz}), 6.68(\mathrm{~d}, 1 \mathrm{H}, J=8.1 \mathrm{~Hz}), 7.17(\mathrm{~d}, 2 \mathrm{H}, J=8.1 \mathrm{~Hz}), 7.42(\mathrm{~d}$, $2 \mathrm{H}, J=8.1 \mathrm{~Hz})$; FT-IR $\left(\mathrm{KBr}, \mathrm{cm}^{-1}\right): 3305,2974,1737,1646,1539,1236$; ESI-MS $(\mathrm{m} / \mathrm{z}, \%): 342,344\left(\mathrm{M}^{+}+\mathrm{H}\right) ;{ }^{13} \mathrm{C}$ NMR $\left(75 \mathrm{MHz}, \mathrm{CDCl}_{3}\right): \delta 17.44,19.47,20.75,23.25$, 29.19, 53.66, 79.53, 121.58, 129.52, 131.40, 137.59, 169.21, 171.19; HRMS for $\mathrm{C}_{15} \mathrm{H}_{20} \mathrm{NO}_{3} \mathrm{NaBr}\left(\mathrm{M}^{+}+\mathrm{Na}\right)$ : calcd. 364.0519, found: 364.0516.

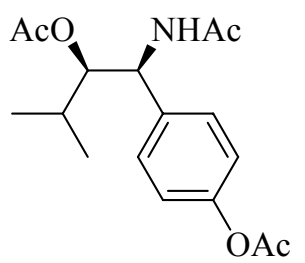

HPLC: Chiralpak AS column; detected at $214 \mathrm{~nm}$, eluent: $n$-hexane/iso-propanol $=90 / 10(\mathrm{v} / \mathrm{v})$.

$[\alpha]_{\mathrm{D}}{ }^{20}=+111.1\left(\right.$ c $\left.0.25, \mathrm{CHCl}_{3}\right) ;{ }^{1} \mathrm{H}$ NMR $\left(300 \mathrm{MHz}, \mathrm{CDCl}_{3}\right): \delta$ $0.91(\mathrm{~d}, 3 \mathrm{H}, J=6.6 \mathrm{~Hz}), 1.00(\mathrm{~d}, 3 \mathrm{H}, J=6.6 \mathrm{~Hz}), 1.79-1.84(\mathrm{~m}$, $1 \mathrm{H}), 1.95(\mathrm{~s}, 3 \mathrm{H}), 1.97(\mathrm{~s}, 3 \mathrm{H}), 2.28(\mathrm{~s}, 3 \mathrm{H}), 4.97(\mathrm{dd}, 1 \mathrm{H}, J=5.4$, $7.2 \mathrm{~Hz}), 5.27(\mathrm{dd}, 1 \mathrm{H}, J=5.4,8.7 \mathrm{~Hz}), 6.22(\mathrm{~d}, 1 \mathrm{H}, J=6.0 \mathrm{~Hz}), 7.04(\mathrm{~d}, 2 \mathrm{H}, J=8.4$ $\mathrm{Hz}), 7.29$ (d, 2H, $J=8.4 \mathrm{~Hz})$; FT-IR $\left(\mathrm{KBr}, \mathrm{cm}^{-1}\right): 3587,3287,1737,1658,1550,1238$; ESI-MS (m/z, \%): $322\left(\mathrm{M}^{+}+\mathrm{H}\right) ;{ }^{13} \mathrm{C}$ NMR $\left(75 \mathrm{MHz}, \mathrm{CDCl}_{3}\right): \delta 17.50,19.45,20.74$, 21.11, 23.23, 29.23, 53.46, 79.64, 121.44, 128.96, 136.03, 149.97, 169.18, 169.44, 171.14; HRMS for $\mathrm{C}_{17} \mathrm{H}_{24} \mathrm{NO}_{5}\left(\mathrm{M}^{+}+\mathrm{H}\right)$ : calcd. 322.1649, found: 322.1646 .

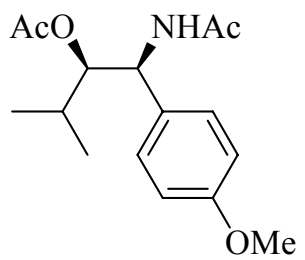

HPLC: Chiralpak AD column; detected at $254 \mathrm{~nm}$, eluent: $n$-hexane/iso-propanol $=90 / 10(\mathrm{v} / \mathrm{v})$.

$[\alpha]_{\mathrm{D}}{ }^{20}=161.2\left(\right.$ c $\left.0.35, \mathrm{CHCl}_{3}\right) ;{ }^{1} \mathrm{H} \mathrm{NMR}\left(300 \mathrm{MHz}, \mathrm{CDCl}_{3}\right): \delta$ $0.91(\mathrm{~d}, 3 \mathrm{H}, J=6.6 \mathrm{~Hz}), 0.98(\mathrm{~d}, 3 \mathrm{H}, J=6.6 \mathrm{~Hz}), 1.76-1.83(\mathrm{~m}$, $1 \mathrm{H}), 1.94(\mathrm{~s}, 3 \mathrm{H}), 1.97$ (s, 3H), $3.79(\mathrm{~s}, 3 \mathrm{H}), 4.98(\mathrm{dd}, 1 \mathrm{H}, J=5.7$, $12.6 \mathrm{~Hz}), 5.20(\mathrm{dd}, 1 \mathrm{H}, J=5.7,8.4 \mathrm{~Hz}), 6.38(\mathrm{~d}, 1 \mathrm{H}, J=8.4 \mathrm{~Hz}), 6.84(\mathrm{~d}, 2 \mathrm{H}, J=8.7$ $\mathrm{Hz}), 7.23$ (d, 2H, $J=8.7 \mathrm{~Hz}$ ); FT-IR $\left(\mathrm{KBr}, \mathrm{cm}^{-1}\right)$ : 3318, 3290, 1746, 1729, 1641, 1517 , 1258; ESI-MS (m/z, \%): $\left.294\left(\mathrm{M}^{+}+\mathrm{H}\right) ;{ }^{13} \mathrm{C} \mathrm{NMR} \mathrm{(75} \mathrm{MHz,} \mathrm{CDCl}_{3}\right): \delta 17.57,19.44$, 20.80, 23.31, 29.26, 53.46, 55.14, 79.86, 113.68, 128.98, 130.51, 158.93, 169.05, 171.10; HRMS for $\mathrm{C}_{16} \mathrm{H}_{23} \mathrm{NO}_{4} \mathrm{Na}\left(\mathrm{M}^{+}+\mathrm{Na}\right)$ : calcd. 316.1519 , found: 316.1519 .

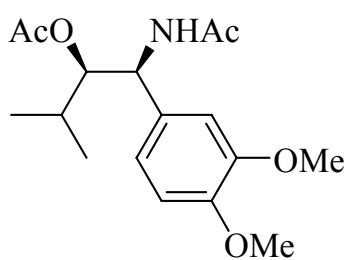

HPLC: Chiralpak AD column; detected at $254 \mathrm{~nm}$, eluent: $n$-hexane/iso-propanol $=90 / 10(\mathrm{v} / \mathrm{v})$.

$[\alpha]_{\mathrm{D}}{ }^{20}=+120.3\left(\right.$ c $\left.0.21, \mathrm{CHCl}_{3}\right) ;{ }^{1} \mathrm{H} \mathrm{NMR}\left(300 \mathrm{MHz}, \mathrm{CDCl}_{3}\right)$ : $\delta 0.88(\mathrm{~d}, 3 \mathrm{H}, J=6.6 \mathrm{~Hz}), 0.93(\mathrm{~d}, 3 \mathrm{H}, J=6.6 \mathrm{~Hz}), 1.77-1.82$ (m, 1H), 1.89 (s, 3H), $1.90(\mathrm{~s}, 3 \mathrm{H}), 3.81(\mathrm{~s}, 3 \mathrm{H}), 4.99(\mathrm{dd}, 1 \mathrm{H}$, $J=6.3,12.6 \mathrm{~Hz}), 5.15(\mathrm{dd}, 1 \mathrm{H}, J=6.6,8.4 \mathrm{~Hz}), 6.42(\mathrm{~d}, 1 \mathrm{H}, J=8.4 \mathrm{~Hz}), 6.75(\mathrm{~d}, 1 \mathrm{H}$, $J=7.8 \mathrm{~Hz}), 6.83(\mathrm{~d}, 2 \mathrm{H}, J=8.4 \mathrm{~Hz})$; FT-IR $\left(\mathrm{KBr}, \mathrm{cm}^{-1}\right): 3328,3271,2933,1749$, 1644, 1521, 1230; ESI-MS (m/z, \%): $324\left(\mathrm{M}^{+}+\mathrm{H}\right) ;{ }^{13} \mathrm{C}$ NMR $\left(75 \mathrm{MHz}, \mathrm{CDCl}_{3}\right): \delta$ 17.26, 19.50, 20.76, 23.25, 29.20, 53.66, 55.69, 55.76, 79.41, 110.83, 111.42, 120.00, 131.09, 148.33, 148.49, 169.10, 170.88; HRMS for $\mathrm{C}_{17} \mathrm{H}_{25} \mathrm{NO}_{5} \mathrm{Na}\left(\mathrm{M}^{+}+\mathrm{Na}\right)$ : calcd. 346.1625, found: 346.1626 . 


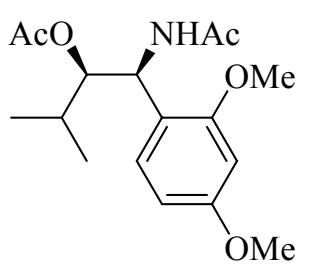

HPLC: Chiralcel OD column; detected at $214 \mathrm{~nm}$, eluent: $n$-hexane/iso-propanol $=95 / 5(\mathrm{v} / \mathrm{v})$.

$[\alpha]_{\mathrm{D}}{ }^{20}=+113.3\left(c 0.20, \mathrm{CHCl}_{3}\right) ;{ }^{1} \mathrm{H}$ NMR $\left(300 \mathrm{MHz}, \mathrm{CDCl}_{3}\right): \delta$ $0.93(\mathrm{~d}, 3 \mathrm{H}, J=6.9 \mathrm{~Hz}), 0.99(\mathrm{~d}, 3 \mathrm{H}, J=6.9 \mathrm{~Hz}), 1.61-1.74(\mathrm{~m}$, $4 \mathrm{H}), 1.75(\mathrm{~s}, 3 \mathrm{H}), 2.18(\mathrm{~s}, 3 \mathrm{H}), 3.79(\mathrm{~s}, 3 \mathrm{H}), 3.88(\mathrm{~s}, 3 \mathrm{H})$, 5.19-5.21 (m, 2H), 6.37-6.44 (m, 2H), $6.63(\mathrm{~d}, 1 \mathrm{H}, J=7.2 \mathrm{~Hz}), 7.06(\mathrm{~d}, 1 \mathrm{H}, J=5.4$ Hz); FT-IR (KBr, cm $\left.{ }^{-1}\right)$ : 3300, 2965, 1741, 1653, 1509, 1239; ESI-MS (m/z, \%): 324 $\left(\mathrm{M}^{+}+\mathrm{H}\right) ;{ }^{13} \mathrm{C}$ NMR $\left(75 \mathrm{MHz}, \mathrm{CDCl}_{3}\right): \delta 15.85,20.05,20.52,23.62,28.49,52.20$, 55.26, 55.57, 77.54, 99.01, 103.92, 118.84, 131.17, 158.56, 160.35, 168.92, 170.36; HRMS for $\mathrm{C}_{17} \mathrm{H}_{25} \mathrm{NO}_{5} \mathrm{Na}\left(\mathrm{M}^{+}+\mathrm{Na}\right)$ : calcd. 346.1625, found: 346.1628 .

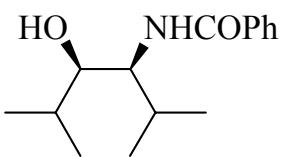

HPLC: Chiralpak AS column; detected at $214 \mathrm{~nm}$, eluent: $n$-hexane/iso-propanol $=95 / 5(\mathrm{v} / \mathrm{v})$.

$[\alpha]_{\mathrm{D}}^{20}=+13.1^{\circ}\left(\right.$ c $\left.0.16, \mathrm{CHCl}_{3}\right) ;{ }^{1} \mathrm{H} \mathrm{NMR}\left(300 \mathrm{MHz}, \mathrm{CDCl}_{3}\right): \delta$ $0.97(\mathrm{~d}, 6 \mathrm{H}, J=3.0 \mathrm{~Hz}), 1.00(\mathrm{~d}, 3 \mathrm{H}, J=3.0 \mathrm{~Hz}), 1.81-1.87(\mathrm{~m}, 1 \mathrm{H}), 2.08(\mathrm{~d}, 1 \mathrm{H}, J$ $=3.3 \mathrm{~Hz}), 2.22-2.28(\mathrm{~m}, 1 \mathrm{H}), 3.41(\mathrm{~d}, 1 \mathrm{H}, J=6.3 \mathrm{~Hz}), 4.20-4.26(\mathrm{~m}, 1 \mathrm{H}), 6.28(\mathrm{~d}, 1 \mathrm{H}$, $J=9.6 \mathrm{~Hz}), 7.40-7.50(\mathrm{~m}, 3 \mathrm{H}), 7.77(\mathrm{~d}, 1 \mathrm{H}, J=6.9 \mathrm{~Hz})$; FT-IR $\left(\mathrm{KBr}, \mathrm{cm}^{-1}\right): 3451$, 3384, 2964, 1641, 1522, 1319; ESI-MS (m/z, \%): $250\left(\mathrm{M}^{+}+\mathrm{H}\right) ;{ }^{13} \mathrm{C}$ NMR $(75 \mathrm{MHz}$, $\left.\mathrm{CDCl}_{3}\right): \delta 16.81,16.97,19.86,21.07,30.33,55.20,77.96,126.83,128.57,131.41$, 134.79, 167.46; HRMS for $\mathrm{C}_{15} \mathrm{H}_{23} \mathrm{NO}_{2} \mathrm{Na}\left(\mathrm{M}^{+}+\mathrm{Na}\right)$ : calcd. 272.1621, found: 272.1615 .

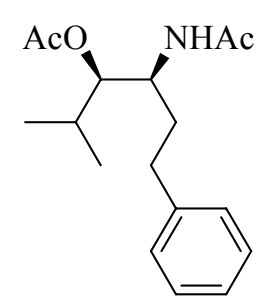

HPLC: Chiralpak AD column; detected at $214 \mathrm{~nm}$, eluent: $n$-hexane/iso-propanol $=95 / 5(\mathrm{v} / \mathrm{v})$.

$[\alpha]_{\mathrm{D}}{ }^{20}=+27.6\left(c 0.11, \mathrm{CHCl}_{3}\right) ;{ }^{1} \mathrm{H}$ NMR $\left(300 \mathrm{MHz}, \mathrm{CDCl}_{3}\right): \delta 0.87$ $(\mathrm{d}, 3 \mathrm{H}, J=2.1 \mathrm{~Hz}), 0.90(\mathrm{~d}, 3 \mathrm{H}, J=2.1 \mathrm{~Hz}), 1.55-1.58(\mathrm{~m}, 1 \mathrm{H})$, 1.81-1.87 (m, 2H), $1.97(\mathrm{~s}, 3 \mathrm{H}), 2.07(\mathrm{~s}, 3 \mathrm{H}), 2.60-2.69(\mathrm{~m}, 2 \mathrm{H}), 4.30$ (t, $1 \mathrm{H}, J=3.3 \mathrm{~Hz}), 4.65(\mathrm{dd}, 1 \mathrm{H}, J=4.5,6.9 \mathrm{~Hz}), 5.72(\mathrm{~d}, 1 \mathrm{H}, J=9.3$ $\mathrm{Hz}), 7.16-7.31$ (m, 5H); FT-IR (KBr, cm $\left.{ }^{-1}\right): 3285,2966,1739,1651,1552,1237$; ESI-MS (m/z, \%): $292\left(\mathrm{M}^{+}+\mathrm{H}\right) ;{ }^{13} \mathrm{C}$ NMR $\left(75 \mathrm{MHz}, \mathrm{CDCl}_{3}\right): \delta 17.87,19.07,20.93$, 23.39, 29.66, 31.57, 32.27, 49.57, 81.19, 125.92, 128.30, 128.39, 141.63, 169.75, 171.44; HRMS for $\mathrm{C}_{17} \mathrm{H}_{25} \mathrm{NO}_{3} \mathrm{Na}\left(\mathrm{M}^{+}+\mathrm{Na}\right)$ : calcd. 314.1727 , found: 314.1728 .

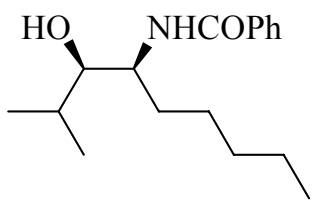

HPLC: Chiralpak AS column; detected at $214 \mathrm{~nm}$, eluent: $n$-hexane/iso-propanol $=95 / 5(\mathrm{v} / \mathrm{v})$.

m.p.:92 ${ }^{\circ} \mathrm{C} ;[\alpha]_{\mathrm{D}}{ }^{20}=-43.9^{\circ}\left(\mathrm{c} 0.15, \mathrm{CHCl}_{3}\right) ;{ }^{1} \mathrm{H}$ NMR $(300 \mathrm{MHz}$, $\left.\mathrm{CDCl}_{3}\right): \delta 0.96(\mathrm{t}, 3 \mathrm{H}, J=6.9 \mathrm{~Hz}), 1.01(\mathrm{~d}, 3 \mathrm{H}, J=7.5 \mathrm{~Hz}), 1.02$ $(\mathrm{d}, 3 \mathrm{H}, J=7.5 \mathrm{~Hz}), 1.29-1.78(\mathrm{~m}, 9 \mathrm{H}), 2.23(\mathrm{~s}, 1 \mathrm{H}), 3.35(\mathrm{~d}, 1 \mathrm{H}, J=7.5 \mathrm{~Hz}), 4.32(\mathrm{t}$, $1 \mathrm{H}, J=7.5 \mathrm{~Hz}), 6.48(\mathrm{~d}, 1 \mathrm{H}, J=8.7 \mathrm{~Hz}), 7.39-7.52(\mathrm{~m}, 3 \mathrm{H}), 7.76-779$ (d, $1 \mathrm{H}, J=7.5$ $\mathrm{Hz}$ ); FT-IR (KBr, cm $\left.{ }^{-1}\right): 3312,2956,1634,1539$; ESI-MS (m/z, \%): $278\left(\mathrm{M}^{+}+\mathrm{H}\right) ;{ }^{13} \mathrm{C}$ NMR $\left(75 \mathrm{MHz}, \mathrm{CDCl}_{3}\right): \delta 14.04,18.90,19.29,22.56,25.87,27.71,31.20,31.84$, $51.56,80.03,126.88,128.52,131.37,134.71,167.16$; HRMS for $\mathrm{C}_{17} \mathrm{H}_{27} \mathrm{NO}_{2} \mathrm{Na}$ $\left(\mathrm{M}^{+}+\mathrm{Na}\right)$ : calcd. 300.1934 , found: 300.1937. 
<smiles>CC(=O)N[C@@H](COc1ccccc1)[C@@H](OC(C)=O)C(C)C</smiles>

HPLC: Chiralcel AD column; detected at $214 \mathrm{~nm}$, eluent: $n$-hexane/iso-propanol $=90 / 10(\mathrm{v} / \mathrm{v})$. $[\alpha]_{\mathrm{D}}{ }^{26}=+17.2\left(\mathrm{c} 3.20, \mathrm{CHCl}_{3}\right) ;{ }^{1} \mathrm{H}$ NMR $\left(300 \mathrm{MHz}, \mathrm{CDCl}_{3}\right) \delta$ 7.35-7.28 (m, 5H), 6.06-6.03 (d, $J=9.0 \mathrm{~Hz}, 1 \mathrm{H}), 4.93-4.89$ (dd, $J_{1}$ $\left.=J_{2}=4.5 \mathrm{~Hz}, 1 \mathrm{H}\right), 4.48-45(\mathrm{~d}, J=6.3 \mathrm{~Hz}, 2 \mathrm{H}), 4.39-4.31(\mathrm{~m}, 1 \mathrm{H}), 3.46-3.44$, 3.46-3.44 (m, 2H), $1.98(\mathrm{~s}, 1 \mathrm{H}), 1.94(\mathrm{~s}, 1 \mathrm{H}), 1.91-1.87(\mathrm{~m}, 1 \mathrm{H}), 0.91(\mathrm{~s}, 3 \mathrm{H}), 0.89(\mathrm{~s}$, $3 \mathrm{H}) ;{ }^{13} \mathrm{C} \mathrm{NMR}\left(75 \mathrm{MHz}, \mathrm{CDCl}_{3}\right) \delta \mathrm{ppm} 170.63,169.51,137.64,128.32,127.88$, 127.72, 76.52, 73.28, 68.43, 48.94, 29.02, 23.32, 20.76, 19.71, 16.41; FT-IR (film $\mathrm{cm}^{-1}$ ) 3282, 2968, 1740, 1652, 1372, 1238; ESI-MS (m/z, \%): $308.2\left(\mathrm{M}^{+}+\mathrm{H}\right), 330.1$ $\left(\mathrm{M}^{+}+\mathrm{Na}\right)$; HRMS for $\mathrm{C}_{17} \mathrm{H}_{25} \mathrm{NO}_{4} \mathrm{Na}\left(\mathrm{M}^{+}+\mathrm{Na}\right)$ : calcd. 330.1676, found: 330.1677 .

\section{Synthesis of D-erythro-sphinganine.}

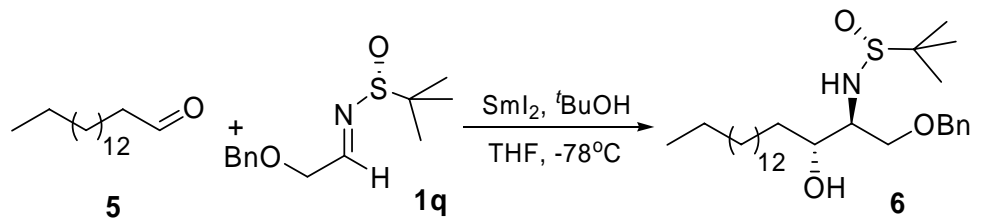

To the solution of $\mathrm{SmI}_{2}(1.0 \mathrm{mmol}$ in $5 \mathrm{~mL}$ of $\mathrm{THF})$ under argon at $-78^{\circ} \mathrm{C}$ was dropped in slowly the solution of imine $1 \mathbf{1 q}(121 \mathrm{mg}, 0.48 \mathrm{mmol})$, palmitaldehyde 5 (450 $\mathrm{mg}$, $2.0 \mathrm{mmol})$ and $t$-Butanol $(95 \mu \mathrm{L}, 1.0 \mathrm{mmol})$ in $10 \mathrm{~mL}$ of THF. The mixture was stirred vigously for $13 \mathrm{~h}$ at the same temperature and then quenched by $1 \mathrm{~mL}$ of saturated $\mathrm{Na}_{2} \mathrm{~S}_{2} \mathrm{O}_{3}$ solution. The organic layer was separated, and the aqueous layer was extracted with ethyl acetate. The combined organic extracts were washed by saturated brine and then dried over anhydrous $\mathrm{MgSO}_{4}$, filtered, and concentrated under vacuum. After flash silica gel chromatography, $19 \mathrm{mg}$ of the starting imine 1q was recovered, and $129 \mathrm{mg}$ (64\% yield based on recovered starting material) of the pure product 6 was obtained as yellow oil. (Please note: Due to the poor solubility of long chained palmitaldehyde in THF, a larger excess (4 equiv) was employed to achieve better result.)

$[\alpha]_{\mathrm{D}}{ }^{26}=-23.9\left(\right.$ c 2.00, $\left.\mathrm{CHCl}_{3}\right) ;{ }^{1} \mathrm{H}$ NMR $\left(300 \mathrm{MHz}, \mathrm{CDCl}_{3}\right) \delta$ 0.88-0.86 (m, 3H), $1.25-1.24(\mathrm{~m}, 35 \mathrm{H}), 1.47-1.40(\mathrm{~m}, 2 \mathrm{H}), 2.49(\mathrm{~d}, J=6.6 \mathrm{~Hz}, 1 \mathrm{H}), 3.33(\mathrm{~m}, 1 \mathrm{H}), 3.69$ $(\mathrm{m}, 1 \mathrm{H}), 3.89-3.74(\mathrm{~m}, 2 \mathrm{H}), 3.99-3.96(\mathrm{~d}, J=8 \mathrm{~Hz}, 2 \mathrm{H}), 4.64-4.44\left(\mathrm{AB}, J_{A B}=11.7 \mathrm{~Hz}\right.$, 2H), 7.36-7.28 (m, 5H); ${ }^{13} \mathrm{C} \mathrm{NMR}\left(75 \mathrm{MHz}, \mathrm{CDCl}_{3)} \delta 137.48,128.50,127.94,127.92\right.$, 73.50, 73.21, 70.10, 59.03, 55.96, 33.83, 31.91, 29.68, 29.67, 29.64, 29.60, 29.57, 29.54, 29.35, 25.83, 22.68, 22.64, 14.13; FT-IR (film cm ${ }^{-1}$ ) 3410 (br, m), 2925, 2855, 1467, 1456, 1073, 1051; MALDI-TOF-MS (m/z, \%): $496\left(\mathrm{M}^{+}+\mathrm{H}\right)$; HRMS for $\mathrm{C}_{29} \mathrm{H}_{54} \mathrm{NO}_{3} \mathrm{~S}\left(\mathrm{M}^{+}+\mathrm{H}\right)$ : calcd. 496.3819, found: 496.3820 .

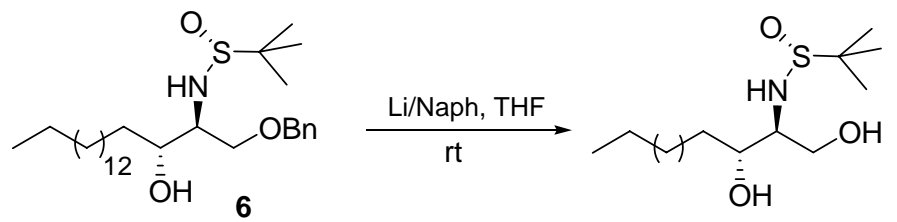


To a solution of naphthalene $(3.5 \mathrm{~g})$ in $20 \mathrm{~mL}$ of THF was added $0.1 \mathrm{~g}$ of Lithium under argon. The mixture was stirred for $2 \mathrm{~h}$ at r.t. to form a dark green solution. This solution was droped into a solution of $6(33 \mathrm{mg}, 0.065 \mathrm{mmol})$ in $2 \mathrm{~mL}$ of dry THF at $0^{\circ} \mathrm{C}$ untill the dark green color did not disappear. The mixture was stirred for $0.5 \mathrm{~h}$ at r.t. then quenched by drops of methanol. The solvent was removed in vacuo and flash silica gel chromatography provided $25 \mathrm{mg}(94 \%)$ of product as colorless needles.

M.p. $41-43^{\circ} \mathrm{C} ;[\alpha]_{\mathrm{D}}{ }^{27}=-17.0\left(\right.$ c $\left.0.55, \mathrm{CHCl}_{3}\right) ;{ }^{1} \mathrm{H}$ NMR $\left(300 \mathrm{MHz}, \mathrm{CDCl}_{3}\right)$ : $\delta$ 0.88-0.84 (m, 3H), 1.50-1.24 (m, 37H), 2.04 (br, 1H), 3.17-3.13 (m, 1H), $3.66(\mathrm{~m}, 1 \mathrm{H})$, 3.95-3.83 (m, 2H), 4.10-4.08 (d, $J=9.3 \mathrm{~Hz}, 1 \mathrm{H}) ;{ }^{13} \mathrm{C}$ NMR $\left(75 \mathrm{MHz}, \mathrm{CDCl}_{3}\right): \delta$ $73.92,62.39,61.91,56.17,34.33,31.90,29.67,29.65,29.64,29.63,29.56,29.54$, 29.34, 25.84, 25.66, 14.10; FT-IR (film cm${ }^{-1}$ ): 3370 (br, s), 2924, 2854, 1468, 1048; ESI-MS (m/z, \%): $406.2\left(\mathrm{M}^{+}+\mathrm{H}\right), 428.1\left(\mathrm{M}^{+}+\mathrm{Na}\right)$; HRMS for $\mathrm{C}_{22} \mathrm{H}_{48} \mathrm{NO}_{3} \mathrm{~S} \quad\left(\mathrm{M}^{+}+\mathrm{H}\right)$ : calcd. 406.3349 , found: 406.3348 .

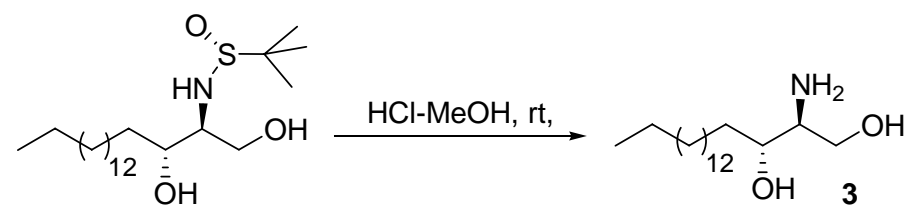

To a solution of the above obtained compound (18 mg, $0.044 \mathrm{mmol})$ in $2 \mathrm{~mL}$ of dry methanol was added the solution of dry $\mathrm{HCl}$ in 1,4-dioxane $(5.8 \mathrm{~N}, 30 \mu \mathrm{L})$. The mixture was stirred at $\mathrm{rt}$ for $0.5 \mathrm{~h}$ and then added in $1 \mathrm{~N} \mathrm{NaOH}$ aqueous solution carefully to adjust the $\mathrm{pH}$ to 9 . Then the solvent was removed in vacuo and flash silica gel chromatography provided $13 \mathrm{mg}$ of the product $3(98 \%)$ as a white solid.

${ }^{1} \mathrm{H}$ NMR $\left(300 \mathrm{MHz}, \mathrm{CDCl}_{3} / \mathrm{MeOH}\right): \delta$ 0.88-0.83 (m, 3H), 1.45-1.24 (m, 28H), 3.04-3.00 (m, 1H), 3.31-3.29 (m, 1H), 3.63-3.59 (m, 1H), 3.69-3.66 (m, 1H), 3.79-3.74 (m, 1H); $\left.{ }^{13} \mathrm{C} \mathrm{NMR} \mathrm{(75MHz,} \mathrm{CDCl}_{3} / \mathrm{MeOH}\right): \delta 71.08,60.06,57.69,33.95$, 32.70, 30.44, 30.30, 30.12, 26.73, 23.40, 14.42; FT-IR (film cm${ }^{-1}$ ): 3359 (br, s), 2924, 2854; ESI-MS (m/z, \%): $302.3\left(\mathrm{M}^{+}+\mathrm{H}\right)$; HRMS for $\mathrm{C}_{18} \mathrm{H}_{40} \mathrm{NO}_{2}\left(\mathrm{M}^{+}+\mathrm{H}\right)$ : calcd. 302.3053, found: 302.3057 .

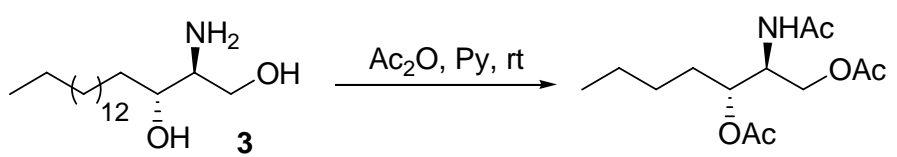

The absolute configuration of $\mathbf{3}$ was further proved by the $[\alpha]_{\mathrm{D}}$ of its triacetate derivative.

$[\alpha]_{\mathrm{D}}^{27}=+17.3\left(c 0.55, \mathrm{CHCl}_{3}\right)\left[\right.$ lit. $[\alpha]_{\mathrm{D}}{ }^{20}=+17.4\left(\right.$ c 1.0, $\left.\mathrm{CHCl}_{3}\right)$ J. Org. Chem. 2000, 65, 3538]; ${ }^{1} \mathrm{H}$ NMR $\left(300 \mathrm{MHz}, \mathrm{CDCl}_{3}\right): \delta 0.88-0.84(\mathrm{~m}, 3 \mathrm{H}), 1.36-1.23(\mathrm{~m}, 26 \mathrm{H})$, 1.59-1.55 (m, 2H), $1.98(\mathrm{~s}, 3 \mathrm{H}), 2.04(\mathrm{~s}, 3 \mathrm{H}), 2.06(\mathrm{~s}, 3 \mathrm{H}), 4.07-4.02(\mathrm{dd}, J=3.6 \mathrm{~Hz}$, $3.9 \mathrm{~Hz}, 1 \mathrm{H}), 4.26-4.20(\mathrm{~m}, 1 \mathrm{H}), 4.39-4.33(\mathrm{~m}, 1 \mathrm{H}), 4.92-4.86\left(\mathrm{AB}, J_{\mathrm{AB}}=6 \mathrm{~Hz}, 1 \mathrm{H}\right)$, $5.43(\mathrm{~d}, J=8.7 \mathrm{~Hz}, 1 \mathrm{H}) ;{ }^{13} \mathrm{C} \mathrm{NMR}\left(75 \mathrm{MHz}, \mathrm{CDCl}_{3}\right): \delta \quad 170.98,170.90,169.74$, $73.91,62.56,50.45,31.87,31.44,29.63,29.60,29.49,29.40,29.31,25.32,23.32$, 22.64, 20.96, 20.78, 14.08; FT-IR (film cm${ }^{-1}$ ): 3302, 2919, 2850, 1734 (s), 1648, 1544; ESI-MS (m/z, \%): $428.2\left(\mathrm{M}^{+}+\mathrm{H}\right), 450.1\left(\mathrm{M}^{+}+\mathrm{Na}\right)$; HRMS for $\mathrm{C}_{24} \mathrm{H}_{45} \mathrm{NO}_{5} \mathrm{Na}\left(\mathrm{M}^{+}+\mathrm{H}\right)$ : calcd. 450.3190 , found: 450.3191 . 


\section{Synthesis of $(3 R, 4 S)$-statine.}

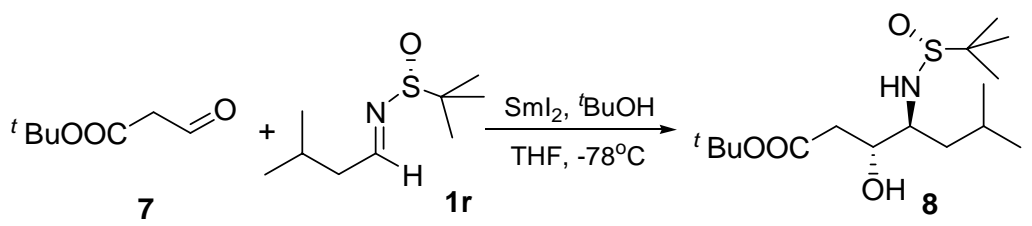

Following the general procedure, 2.0 equiv of aldehyde 7 was used in this reaction, and the reaction mixture was stirred for $6 \mathrm{~h}$. After general workup, the crude product was purified by column chromatography on silica gel to give 8 in $58 \%$ yield.

$[\alpha]_{\mathrm{D}}{ }^{20}=-85\left(c 0.11, \mathrm{CHCl}_{3}\right) ;{ }^{1} \mathrm{H} \mathrm{NMR}\left(300 \mathrm{MHz}, \mathrm{CDCl}_{3}\right): \delta 0.85(\mathrm{~d}, 3 \mathrm{H}, J=6.6 \mathrm{~Hz})$, $0.88(\mathrm{~d}, 3 \mathrm{H}, J=6.6 \mathrm{~Hz}), 1.15-1.35(\mathrm{~m}, 10 \mathrm{H}), 1.43(\mathrm{~s}, 9 \mathrm{H}), 1.53-1.58(\mathrm{~m}, 1 \mathrm{H})$, 1.73-1.76 (m, 1H), 2.29-2.48 (m, 2H), 3.28-3.36 (m, 1H), $3.37(\mathrm{~d}, 1 \mathrm{H}, J=1.2 \mathrm{~Hz})$, $3.66(\mathrm{~d}, 1 \mathrm{H}, J=3.9 \mathrm{~Hz}), 3.95(\mathrm{dd}, 1 \mathrm{H}, J=4.5,8.4 \mathrm{~Hz})$; FT-IR $\left(\mathrm{KBr}, \mathrm{cm}^{-1}\right): 3290$, 2962, 1722, 1366, 1157, 1028; ESI-MS (m/z, \%): $336\left(\mathrm{M}^{+}+\mathrm{H}\right) ;{ }^{13} \mathrm{C}$ NMR $(75 \mathrm{MHz}$, $\left.\mathrm{CDCl}_{3}\right): \delta 21.21,22.66,23.50,23.84,27.96,38.10,38.35,55.87,58.32,71.39,81.20$, 171.77; HRMS $\mathrm{C}_{16} \mathrm{H}_{33} \mathrm{NO}_{4} \mathrm{SNa}\left(\mathrm{M}^{+}+\mathrm{Na}\right)$ : calcd. 358.2022, found: 358.2022 .

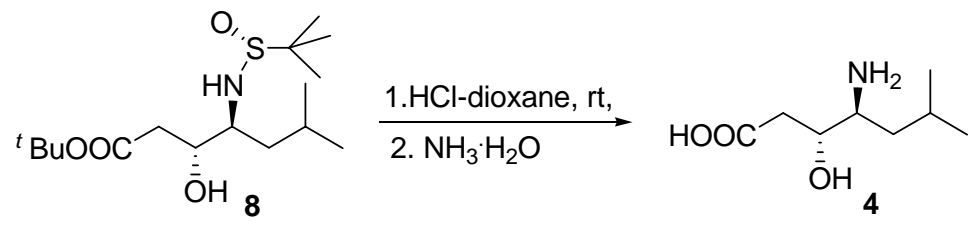

To the solution of $\mathrm{HCl}$ in dioxane $(5.8 \mathrm{~N}, 0.5 \mathrm{~mL})$ was added the coupling product (42 $\mathrm{mg}, 0.125 \mathrm{mmol}$ ) and stirred at $\mathrm{rt}$ for $5 \mathrm{~h}$. The reaction solution was washed with ether; the separated aqueous layer was neutralized with ammonia. After concentration under reduced pressure, the resulting residue was purified by preparative TLC (silica gel) to give $18 \mathrm{mg}$ ( $80 \%$ yield $)$ of $(3 R, 4 S)$-statine.

$[\alpha]_{\mathrm{D}}{ }^{20}=-18.6\left(c 0.08, \mathrm{H}_{2} \mathrm{O}\right)\left[\right.$ lit. $[\alpha]_{\mathrm{D}}{ }^{20}=-19\left(c 0.73, \mathrm{H}_{2} \mathrm{O}\right)$; For $(3 S, 4 R)$-statine, $[\alpha]_{\mathrm{D}}{ }^{20}$ $=+18\left(c 0.88, \mathrm{H}_{2} \mathrm{O}\right)$, J. Med. Chem. 1979, 22, 577]; ${ }^{1} \mathrm{H}$ NMR $\left(300 \mathrm{MHz}, \mathrm{D}_{2} \mathrm{O}\right): \delta 0.77$ $(\mathrm{d}, 3 \mathrm{H}, J=6.6 \mathrm{~Hz}), 0.82(\mathrm{~d}, 3 \mathrm{H}, J=6.6 \mathrm{~Hz}), 1.30-1.38(\mathrm{~m}, 2 \mathrm{H}), 1.51-1.55(\mathrm{~m}, 1 \mathrm{H})$, $2.32(\mathrm{t}, 1 \mathrm{H}, J=4.8 \mathrm{~Hz}), 3.29-3.32(\mathrm{~m}, 1 \mathrm{H}), 4.11-4.13(\mathrm{~m}, 1 \mathrm{H})$; FT-IR $\left(\mathrm{KBr}, \mathrm{cm}^{-1}\right)$ : 2961, 2871, 1721, 1183; ESI-MS (m/z, \%): $176.2\left(\mathrm{M}^{+}+\mathrm{H}\right)$; $\mathrm{HRMS} \mathrm{C}_{8} \mathrm{H}_{18} \mathrm{NO}_{3}\left(\mathrm{M}^{+}+\mathrm{H}\right)$ : calcd. 176.1281, found: 176.1284 . 